\title{
Admittance Control for Robotic Loading: Design and Experiments with a 1-Tonne Loader and a 14-Tonne LHD
}

\author{
Andrew A. Dobson* \\ Mining Systems Laboratory \\ Queen's University \\ Kingston, ON K7L 3N6 Canada \\ andrew.dobson@queensu.ca
}

\author{
Joshua A. Marshall ${ }^{\dagger}$ \\ Mining Systems Laboratory \\ Queen's University \\ Kingston, ON K7L 3N6 Canada \\ joshua.marshall@queensu.ca
}

\author{
Johan Larsson \\ Division Rocktec Automation \\ Atlas Copco Rock Drills AB \\ SE-701 Örebro, Sweden \\ johan. larsson@se.atlascopco.com
}

\begin{abstract}
This paper describes the design, tuning, and extensive field testing of an admittance-based Autonomous Loading Controller (ALC) for robotic excavation. Several iterations of the ALC were tuned and tested in fragmented rock piles - similar to those found in operating mines - by using both a robotic 1-tonne capacity Kubota R520S diesel-hydraulic surface loader and a 14-tonne capacity Atlas Copco ST14 underground load-haul-dump (LHD) machine. On the R520S loader, the ALC increased payload by $18 \%$ with greater consistency, although with more energy expended and longer dig times when compared with digging at maximum actuator velocity. On the ST14 LHD, the ALC took $61 \%$ less time to load $39 \%$ more payload when compared to a single manual operator. The manual operator made $28 \mathrm{dig}$ attempts by using three different digging strategies, and had one failed dig. The tuned ALC made $26 \mathrm{dig}$ attempts at 10 and $11 \mathrm{MN}$ target force levels. All $1011 \mathrm{MN}$ digs succeeded while 6 of the $1610 \mathrm{MN}$ digs failed. The results presented in this paper suggest that the admittance-based ALC is more productive and consistent than manual operators, but that care should be taken when detecting entry into the muck pile.
\end{abstract}

\section{INTRODUCTION}

Robotic diesel-hydraulic Load-Haul-Dump (LHD) machines, such as the Atlas Copco ST14 shown in Figure 1, can haul and dump autonomously (e.g., see Marshall, Barfoot, \& Larsson, 2008), but still require an operator to load the bucket with fragmented rock. Fully automating the LHD cycle could reduce operating costs and improve efficiency by allowing operators to supervise LHDs, rather than providing the real-time commands necessary to perform loading. Automating LHDs is also attractive because the LHD cycle is repetitive,

\footnotetext{
*Now with Clearpath Robotics Inc., Kitchener, ON N2R 1H2 Canada, +1 800-301-3863.

${ }^{\dagger}$ Corresponding author; https://msl.engineering.queensu.ca, +1 613-533-2921.
} 


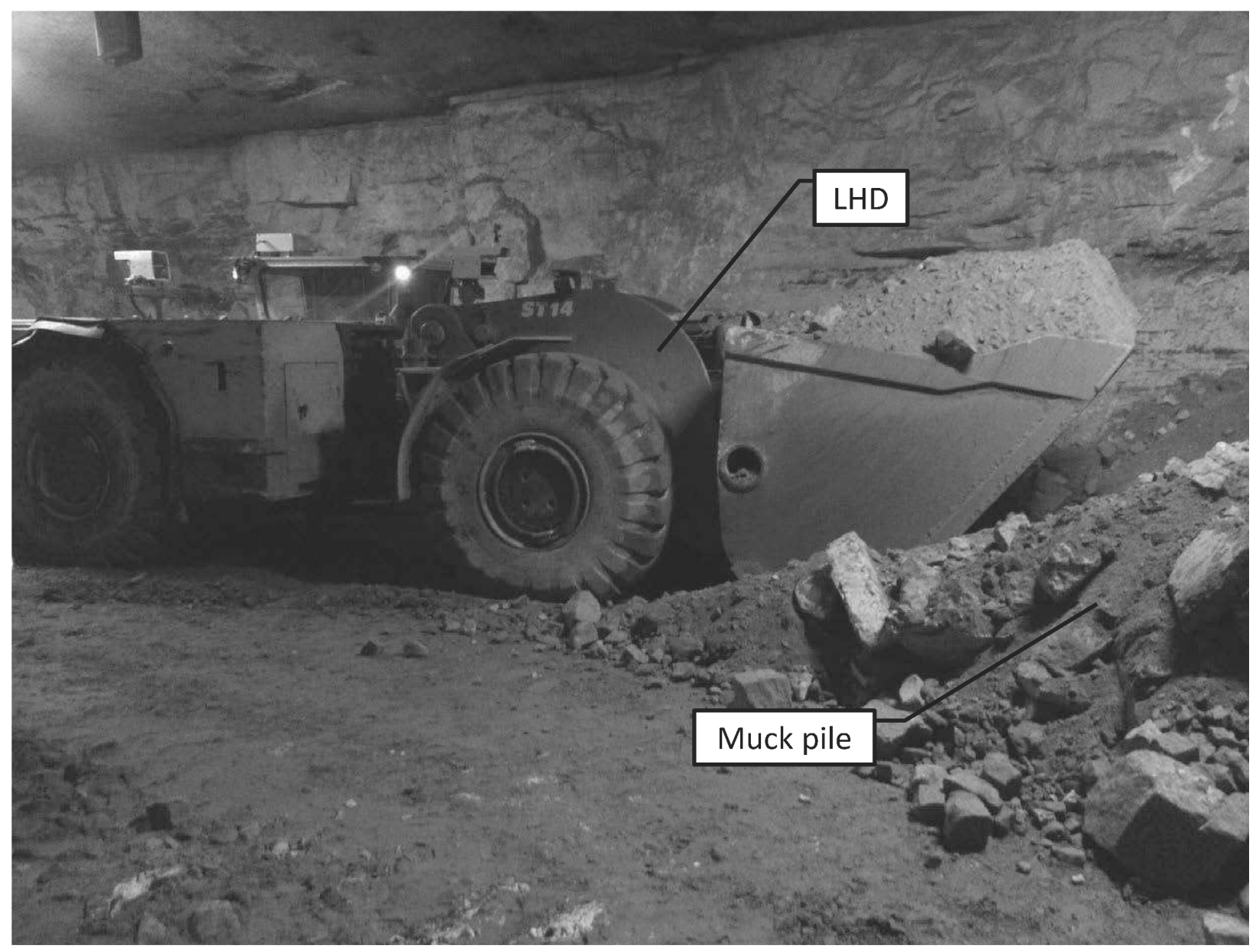

Figure 1: Atlas Copco's Scooptram ST14 load-haul-dump (LHD) vehicle is equipped with an autonomous tramming system that can autonomously haul and dump fragmented rock in underground mines (Marshall, Barfoot, \& Larsson, 2008).

and mining takes place in relatively hazardous, unpleasant environments. Minimizing worker exposure to underground mine environments is also desirable due to the costs associated with keeping operators safe and comfortable in these environments (Zlotnikov, 2012).

The robotic loading algorithm described in this paper uses admittance control to regulate the motion of the vehicle's bucket through a pile of fragmented rock (in mining, also called a muck pile) such that the bucket is filled by the end of the dig. An admittance-based loading controller takes in resistance from the rock pile as an input, and outputs changes in bucket velocity. While one specific version of this approach was proposed for robotic loading by Marshall, Murphy, and Daneshmend (2008), it was never implemented nor was it tested. In this paper, we first report on the development and experimental field testing of an admittance-based autonomous loading controller (ALC), which was implemented on a robotic 1-tonne capacity Kubota R520S surface loader. We then describe extensive field trials of an admittance-based ALC applied to a robotic 14-tonne capacity Atlas Copco ST14 LHD machine at an underground mine near Örebro, Sweden. An abbreviated version of 
these ST14 results was published by Dobson, Marshall, and Larsson (2015).

To the best of our knowledge, this is the first time an admittance controller has been implemented for robotic loading in rock piles with a large rock size distribution on industrial scale vehicles. We were able to demonstrate superior payload mass and greater dig efficiency consistency over digging at maximum bucket velocity (on the R520S) - which is similar to the manual loading results presented by Marshall, Murphy, and Daneshmend (2008) —and over the performance of manual operators (on the ST14).

Robotic excavation involves both dig planning and autonomous loading. This paper focuses on the autonomous loading problem in fragmented rock, as it pertains to loading LHD machines at underground drawpoints. In some cases, the planning problem may be simplified because material flows into a constrained space and there is no need to re-organize the pile or make other high-level planning decisions. However, this may not always be the case. Yet, given an effective autonomous loading algorithm, one could foreseeably develop more sophisticated planning strategies.

\section{BACKGROUND}

Robotic excavation includes both high-level dig planning, and low-level loading. Dig planning involves determining where and how the vehicle will approach and shape the rock pile, while loading involves determining the bucket motions that will be used to fill the bucket. The work described in this paper focuses primarily on the loading problem. However, both topics are briefly reviewed in this section to properly frame the loading problem.

\subsection{Dig Planning}

The dig planning literature is dominated by surface loading and trenching because the final pile or trench geometry is usually more important than filling the digging implement. Petty, Billingsley, and Tran-Cong (1997) proposed linking the rock size distribution in the rock pile to optimum digging trajectories, but this link was never determined. Singh (1995) created a dig planner that selects actions from the action space of a robotic arm excavating sand. His planner found the best action for the current sensed state by using a goal function and an exhaustive search of the action space. Although pioneering work, to the best of our knowledge, this method was never directly applied to randomly fragmented rock.

Thangavelautham (2008), and Fu (2011) proposed an action-based neural network controller, which evolves complex plans from simple actions. In practice, work needs to be done to minimize neural network training time to ensure that solutions can be found quickly when the work environment changes. Neural networks, in general, can suffer from an inability to generalize beyond their training dataset. For robotic loading, this lack of predictability can only be partially overcome by training controllers extensively in a wide variety of environments. 
During the excavation of fragmented rock, there may be instances where some rock fragments exceed the size of the bucket (in mining, this is called oversize). A planning algorithm may attempt to identify and circumvent any detectable oversize material until it can be dealt with by other means. This problem was recently studied by McKinnon and Marshall (2014), where a time-of-flight camera was used to estimate the location and size of potentially oversize material. Once characterized, these areas could be avoided by altering the entry point of an autonomous loading algorithm.

\subsection{Autonomous Loading}

Over many years, several researchers have attempted to automate the loading process. Relatively comprehensive reviews can be found in Marshall (2001); Hemami and Hassani (2009). However, to the best of our knowledge and at the time of writing, no consistently reliable or commercially deployed system yet exists for robotic excavation in fragmented rock, which is the most common target medium for mining applications.

\subsubsection{Scripted Methods}

Sarata, Koyachi, and Sugawara (2008), Almqvist (2009), Shull (2012), and Rocke (1996) all used scripted paths or bucket rates to excavate both gravel and general targets. Blackwell (2013) also used an operator's last dig path as the script for an open pit cable shovel excavating fragmented rock. The methods by Sarata et al. (2008), Almqvist (2009), and Rocke (1996) use the digging forces or pressures to shift between preplanned discrete dig paths. Sarata et al. (2008) and Almqvist (2009) used these algorithms to excavate gravel rock piles, while Caterpillar's patents - Shull (2012) and Rocke (1996) - specify more general targets. No data was given to support the effectiveness of Caterpillar's patents, but their similarity to the methods of Sarata et al. (2008) and Almqvist (2009) means they would likely suffer from the same inability to handle subsurface obstacles. The patent described in Rocke (1996) commands the bucket to move at its maximum velocity as soon as the forces exceed an entry target force. As the experiments in this paper demonstrate, while it is possible to autonomously load by using maximum bucket curl, this approach usually results in poor and highly variable bucket filling. In this paper, we describe a method that dynamically alters the dig path when the digging forces change in response to varying conditions within the pile. We show in Sections 5 and 6 that this adaptability tends to increase both payload and payload consistency.

\subsubsection{Lookup Tables}

Caterpillar Inc. has two additional patents that attempt to alter the dig path by using lookup tables and the dig forces. The first patent (Rocke, 1997) proposes using shovel actuator pressures or forces to select actuator velocities from a table of historical dig data. Caterpillar Inc. proposed a similar lookup table algorithm for drive train and actuator velocity con- 
trollers in a second patent (Alshaer, Ingram, Krone, Berry, \& Harris, 2009). In this later embodiment, a fuzzy logic selector dictates target velocities for the drive train and actuator velocity controllers. While they suggest that an algorithm could be used to select desired velocities, they appear to only select maximum and minimum velocities given the current velocities and digging phase. Although these two approaches are more flexible than their previous patent (Rocke, 1996), they likely require a great deal of testing and tuning to obtain the table of force/velocity or velocity/velocity relationships that result in effective digging. These relationships are also likely to change frequently due to the unstructured nature of the loading problem.

\subsubsection{Behaviour-Based Methods}

Other researchers have proposed dealing with these irregular relationships by using behaviour-based controllers. Lever (2001) — based on earlier work by Shi, Lever, and Wang (1998) - used a fuzzy logic behaviour-based controller to excavate surface rock piles with a 20-tonne surface loader. While they were able to show good results when compared to an expert operator, the algorithm was not as consistent as the expert operator in dig time and final payload. From an engineering perspective and in our opinion, fuzzy behaviour-based controllers are generally less desirable than more conventional controllers because they often perform unpredictably in environments that differ from their training environment, and thus can be challenging to implement and service as a commercial product.

Dasys, Geoffroy, and Drouin (1999) employed fuzzy logic behaviour-based controllers to excavate surface muck piles by using realistic mining equipment. While this group reported a $9 \%$ payload improvement over experienced operators in a real underground mine setting, there was no discussion about payload consistency. The implied need for the operator to configure the controller for each dig target suggests an involved tuning process. There is no known product associated with this patent.

Schmidt, Proetzsch, and Berns (2010) used a simulated 20-tonne Volvo EW/180B bucket excavator to shape soils for construction and landscaping using Integrated Behaviour Based Control (IB2C). IB2C architectures use standardized interfaces for all behaviours and behaviour selectors, which in theory makes them more predictable and adaptable. They used a spherical particle physics simulation to develop all behaviours and behaviour selection controllers. While their IB2C algorithm demonstrated simulated disturbance rejection, no real-world data was collected.

Bradley and Seward (1998) used a one-fifth scale model of a backhoe arm and eventually a JCB801 $360^{\circ}$ tracked excavator to excavate soils by using what they refer to as a behaviourbased Artificial Intelligence (AI) controller. The behaviours use velocity controllers to control the actuators, and observers to infer the digging forces from the velocity tracking error. The AI portion of this controller uses over 80 heuristically derived rules to select a given behaviour, and the only prescribed behaviours are the initial extension and entry behaviours. The controller compares the trajectory of each pass to the current pass and continues excavating 
until the desired trench geometry is achieved. While they say they achieved similar results to expert operators, the final automated path error was never compared to a manual path error. This type of system might also be difficult to implement and service as a product.

These behaviour-based methods highlight the value of using the dig reaction forces to sense the hidden environment within the pile, and the importance of intelligently altering the bucket velocity when obstacles are encountered. They are however, sometimes difficult to derive, predict, operate, and service. In contrast, we contend that conventional force controllers are more predictable than these behaviour-based methods (which may exhibit inconsistent behaviour on different runs) and they do away with fuzzy training approaches in favour of more systematic controller modelling and tuning procedures.

\subsubsection{Impedance Control}

One well-established and widely employed force control method is impedance control. Impedance controllers are used to control the relationship between the tool path and the force experienced by the tool, instead of controlling both the path and reaction forces directly (Spong \& Vidyasagar, 1989). With impedance control, the target is modelled as an admittance and the robot is modelled as an impedance (Bernold, 1993). The measured force $f_{S}$ and sensed path (in terms of actuator velocity) $v_{a}$ are related to the mechanical impedance $Z$ by

$$
Z=\frac{f_{S}}{v_{a}}
$$

For example, an impedance controller for the system could be specified as

$$
f=k_{d} \cdot \frac{d}{d t} e_{v}+k_{p} \cdot e_{v}+k_{i} \cdot \int_{0}^{t} e_{v} d t,
$$

where $k_{d}, k_{p}$, and $k_{i}$ are gains that dictate the impedance relationship between the actuator and the environment.

Richardson-Little and Damaren (2008) used a compliance-based controller to dig through soil targets. Similarly, Bernold (1993) used a sub-scale robot arm, and an impedance controller to dig through dry and wet sand. These controllers were only tested in relatively homogeneous materials, and they did not perform well when they encountered sub-surface obstacles. Hence they are likely poorly suited for autonomously loading fragmented rock piles.

Ha, Santos, Nguyen, Rye, and Durrant-Whyte (2002) and Salcudean, Tafazoli, HashtrudiZaad, and Lawrence (1998) used impedance controllers and small hydraulic shovels to excavate soils. Their impedance controllers tracked the dig path, while low level PD controllers controlled the hydraulic actuators. They used a simple soil model to estimate the digging forces the loader encountered, and these forces were used to alter the dig trajectory according to the dynamics of the impedance controllers. Unfortunately they did not establish performance criteria like our dig efficiency metric (defined in Section 5.2), and the path history they did publish seems to have poor convergence between the target path and the actual 
bucket path. This divergence could be due to encountering subsurface areas with high force that matches or exceeds the force commands sent to the actuators. When this force relationship exists, it becomes impossible for the impedance controller to further reduce the tracking error. This problem was addressed by Maeda, Rye, and Singh (2013) by including a torque observer in their impedance controller.

Maeda et al. (2013) used a torque based impedance controller to excavate soils by using a Komatsu PC05-7 1.5-tonne excavator. Their controller uses a torque observer to overcome the high $f_{S}$, low correction force problem experienced by Salcudean et al. (1998) by augmenting the controller torque commands when high forces are detected. Their controller uses a feed forward friction torque estimator to remove the non-linear friction torques and compensate for the high gains necessary to control the low bandwidth excavator. While their controller was successful and overcame subsurface obstacles by using the observer, they decided to use an impedance controller because the final trench geometry was more important than filling the bucket. In underground mining, the bucket path is almost irrelevant because new material is regularly added to the muck pile as it "flows" from a loading point. This realization led Marshall, Murphy, and Daneshmend (2008) to propose that an admittance controller may be better suited to the loading problem in fragmented rock.

\subsubsection{Admittance Control for Loading}

The paper by Seraji (1994) introduces admittance control (but not in the context of robotic loading) and proposes an adaptive admittance controller to improve controller response when environmental stiffness changes abruptly. Seraji (1994) used a serial robot with simulated environment stiffness to evaluate this adaptive admittance control scheme. Mechanical admittance $Y$ is defined as

$$
Y=\frac{v_{a}}{f_{S}}
$$

where $v_{a}$ is the actuator velocity, and $f_{S}$ is the environment reaction force loading the actuator. In a basic admittance controller, an admittance model $Y$ is selected to modulate the error $e_{f}$ between the target force $f_{T}$ and the sensed force $f_{S}$; i.e., $e_{f}=f_{T}-f_{S}$. Hence, a general admittance control structure is

$$
v=Y\left(e_{f}\right)
$$

For example, by assuming a second order admittance model, the desired actuator velocity could be given by

$$
v=k_{d} \cdot \frac{d}{d t} e_{f}+k_{p} \cdot e_{f}+k_{i} \cdot \int_{0}^{t} e_{f} d t
$$

where $k_{d}, k_{p}$, and $k_{i}$ are gains that dictate the admittance relationship between the actuator and the environment.

Simply put, mechanical impedances are systems that accept motion and respond with force, while admittances are systems that accept force and respond with motion. Marshall, Murphy, 

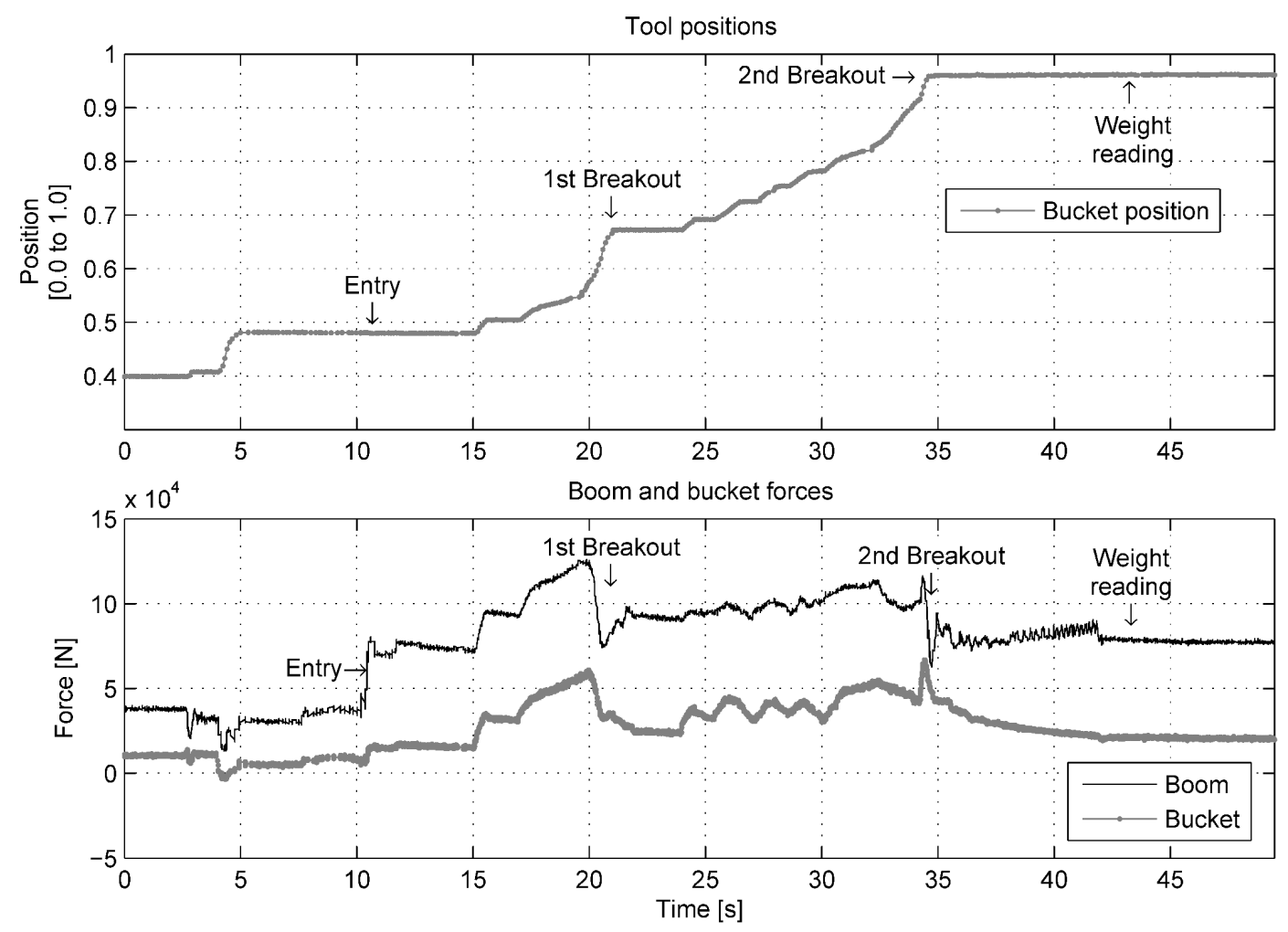

Figure 2: Example robotic dig attempt using the apparatus described in Section 4 and an admittance-based controller (described in Section 3). Three key digging phases (entry, digging, and breakout) can be identified by abrupt changes in hydraulic actuator forces and bucket position. First breakout occurs when the bucket motion causes substantial rock motion, and true breakout occurs after the bucket has curled so much that further penetration is impossible. Rock in the bucket is weighed after the boom and bucket come to rest.

and Daneshmend (2008) and Marshall (2001) suggested using admittance control for the loading problem. Under this framework, the loader is viewed as an admittance, rather than an impedance. They came to this realization after characterizing the digging control signals commanded by expert operators loading in fragmented rock.

For example, in this paper, a series of similar dig trials were performed by using a 1-tonne surface loader and a pile of fragmented limestone. Figure 2 shows a typical bucket position and hydraulic actuator force profile from an autonomous dig attempt (as described in Section 3). Both the actuator position, and the resulting forces can be used to define three distinct dig phases: (1) entry, (2) digging, and (3) breakout. At entry, the forces rise sharply. During digging, the algorithm reduces the sensed forces by moving the bucket, which moves the rock surrounding the bucket. This relationship was also observed in the manual loading data set collected by Marshall (2001). Finally breakout occurs when the forces drop off suddenly indicating that the bucket has lost contact with the surrounding rock.

Johnson, Sahlin, Anderson, and Cherney (2010) recently proposed an admittance-like con- 


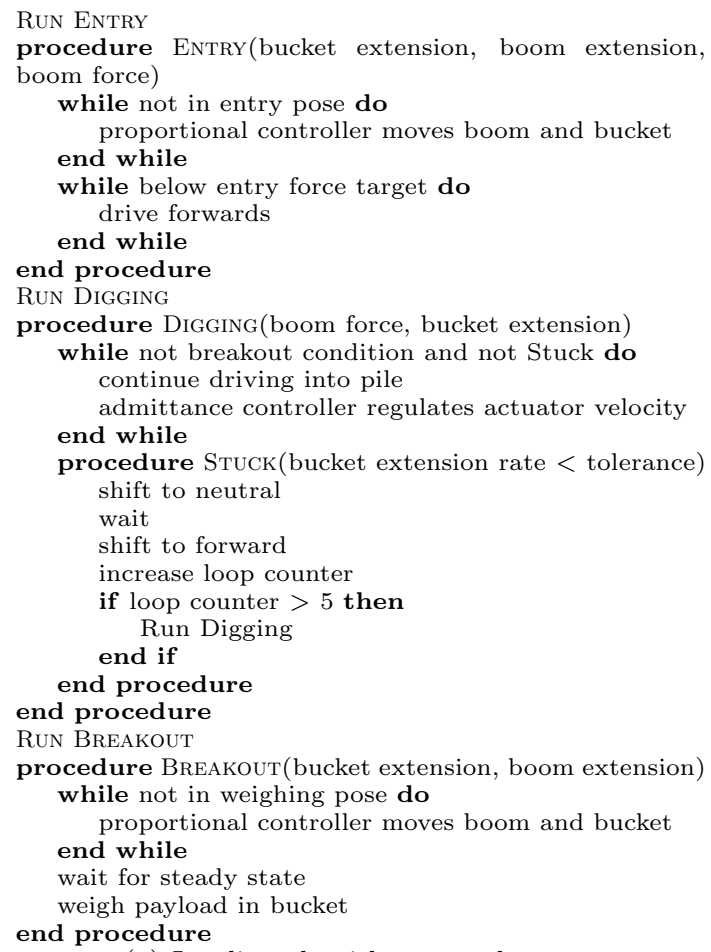

(a) Loading algorithm procedures

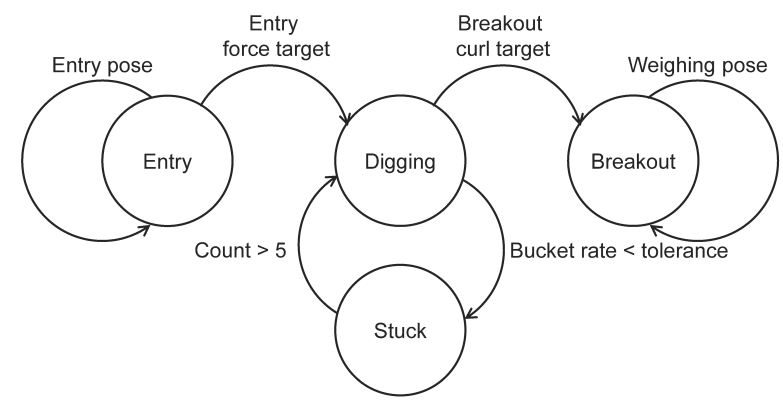

(b) Finite state machine representation

Figure 3: (a) The ALC algorithm has three main parts: entry, digging, and breakout. In this algorithm, 'driving forward' means steering is locked, loader gearshift is set to forward, throttle is set to full, and partial brake is applied to limit forward thrust. (b) If the bucket actuator extension velocity approaches a velocity tolerance the stuck condition is triggered.

troller that uses wheel torque to modulate actuator velocity. They use two desired wheel torque levels (depending on boom position) to proportionally alter the velocity of the boom and bucket. While this method is similar to an admittance controller, wheel torque is very challenging to measure, especially since wheel slip is so common in mining applications.

\section{AUTONOMOUS LOADING CONTROLLER}

An overview of the autonomous loading controller (ALC) used in the experiments presented by this paper is shown in Figure 3. It has three main parts, which correspond to the three major dig phases. These three dig phases are shown schematically in Figure 4. The most critical part of the loading algorithm is the admittance controller, which governs the motion of the bucket through the pile. The admittance controller uses the forces sensed in the boom actuator to control the velocity of the bucket actuator. One advantage of this method is that a model is not required to predict the motion of the excavator and the muck pile. For a more detailed treatment of excavator kinematics please see the fundamental work by Hemami and Daneshmend (1992). Three admittance controllers were used in the field experiments described in this paper, as discussed in Section 3.1. 


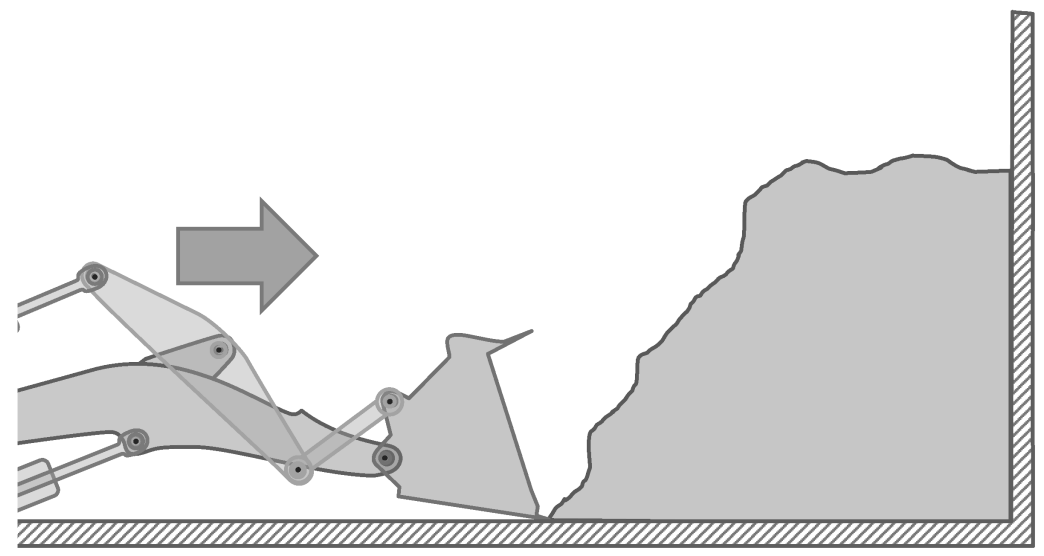

(a) Entry

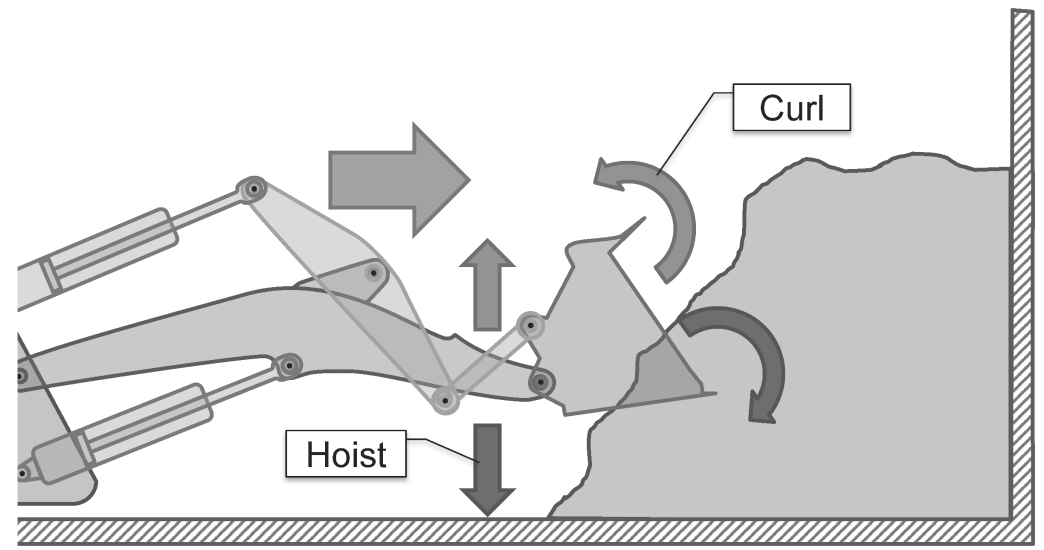

(b) Digging

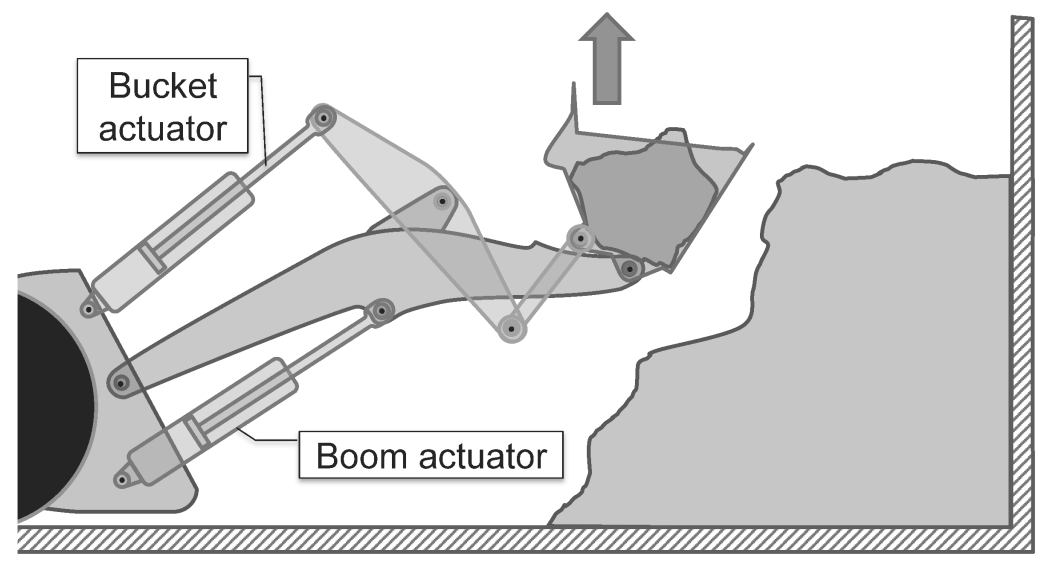

(c) Breakout

Figure 4: (a) Entry ends when the bucket is in the entry position, and the forward motion of the vehicle causes the bucket rock reaction forces to rise above a preset value. (b) During digging, the admittance controller causes the bucket to curl upwards or downwards to maintain a desired reaction force while the boom position is maintained to measure the digging reaction forces. (c) Breakout starts when the bucket has fully curled, which is more reliable than detecting the force drop, and ends when the boom has hoisted to the weighing position. 


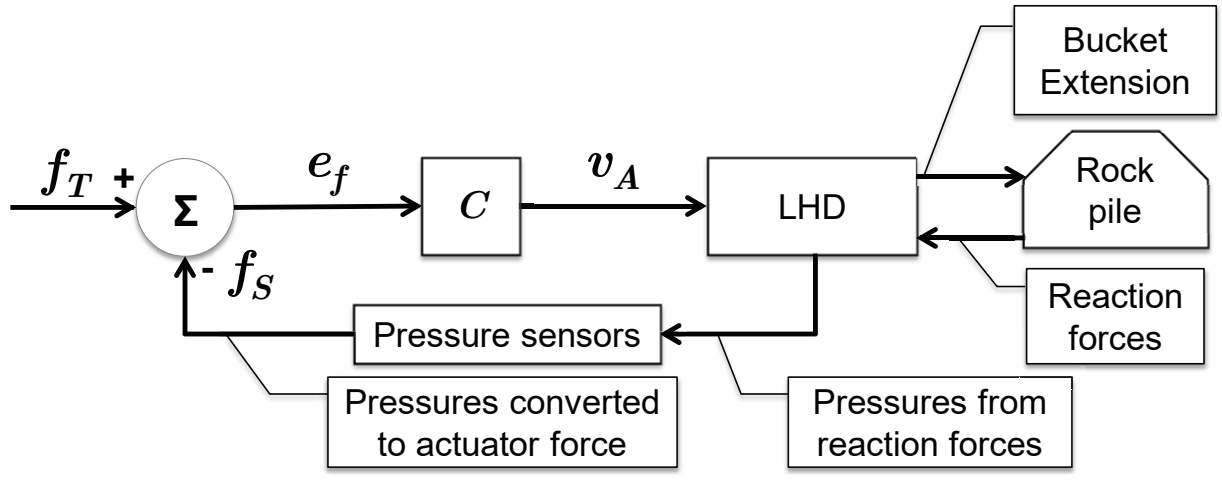

Figure 5: An admittance-based robotic loading algorithm may use any suitable controller $C$ to map the error between the setpoint and sensed actuator forces to the range of possible actuator velocities.

\subsection{Admittance Controller Designs}

The admittance controller designs used in our field experiments were tested by using the error $e_{f}$ between a digging force target $f_{T}$, and the sensed dig reaction forces $f_{S}$ to alter the retraction velocity $v_{A}$ of the bucket actuator. The force $f_{S}$ was calculated by measuring the rod pressure $P_{r}$ and the cylinder pressure $P_{c}$ in either the boom or the bucket actuator. Assuming quasi-static conditions, and given the rod and cylinder cross sectional areas $A_{r}$ and $A_{c}$, the force $f_{S}$ is given by

$$
f_{S}=P_{c} A_{c}-P_{r} A_{r}
$$

A generalized block diagram for admittance-based excavation is shown in Figure 5. Any controller $C$ can be used to map $e_{f}$ to $v_{A}$. Although several cases were studied, this paper presents three specific cases together with corresponding field experiments that employ two different machines and operating scenarios.

In Case 1 (Section 3.2), a one-sided Proportional (P) admittance controller was studied, where $f_{T}$ was set relatively low and the bucket curled back (i.e., $v_{A}>0$ ) when $f_{S}$ in the loader's boom actuator is above $f_{T}$. In Case 2 (Section 3.3), a double-sided Proportional Integral (PI) controller was studied, where $f_{T}$ was relatively high and $v_{A}>0$ when $f_{S}$ in the loader's boom actuator was below $f_{T}$. After working on Cases 1 and 2, Case 3 (Section 3.4) was developed to study a two-sided $\mathrm{P}$ admittance controller, where $f_{T}$ was set relatively high. Cases 1 and 2 were tested in the field on a robotic Kubota R520S, while Case 3 was tested underground on an Atlas Copco ST14 LHD. In this section we present the specifics of each design, while the results of the corresponding experiments are described in Section 5.

In order for an autonomous dig to complete, $f_{T}$ must be set such that $C$ biases the bucket towards curling back (i.e., $v_{A}>0$ ). This bias helps ensure that the breakout condition will eventually be met. If perfect tracking occurs (i.e., $e_{f}$ and $v_{A} \rightarrow 0$ ), then the loader will clearly never reach the breakout condition (cf. Figure 2). Thus, selection of an appropriate $f_{T}$ plays a key role in the design of $C$, as is illustrated by the following two cases. 


\subsection{Case 1 (R520S): One-Sided Proportional Control}

In this case, a one-sided proportional version of the admittance controller is given by

$$
v_{A}= \begin{cases}-k_{p} e_{f} & \text { if } e_{f} \leq 0 \\ 0 & \text { if } e_{f}>0\end{cases}
$$

where $v_{A}$ is the actuator velocity, $k_{p}>0$ is the admittance proportional gain, and the force error $e_{f}$ is given by

$$
e_{f}=f_{T}-f_{S}
$$

For this case and for the loader and muck pile used in the experiments described in Section 4, $f_{T}$ was set at $80 \mathrm{kN}$ (which is almost $100 \mathrm{kN}$ lower than the $f_{T}$ used in Case 2), while $k_{p}$ was set at $1.1 \times 10^{-7} \cdot f_{T}>f_{S}$ results in positive $e_{f}$ and no motion due to the condition specified in Equation (7). $f_{T}<<f_{S}$ will result in high negative $e_{f}$ and controller saturation once the bucket actuator reaches its maximum velocity. The $\mathrm{P}$ controller is one-sided to prevent downward curl since downward curl tends to lift the front wheels, which dramatically reduces forward thrust. This controller has two failure modes (aside from low entry forces not triggering the entry force condition in Figure 3(b)): 1) $e_{f}$ can be low at entry resulting in low $v_{A}$ precisely when rapid curling is more desirable, and 2) $f_{S}$ can be reduced close to $f_{T}$ before breakout and the dig can stall because $e_{f} \rightarrow 0$ and $v_{A} \rightarrow 0$. The PI controller in Case 2 circumvents these failure modes by using a high $f_{T}$ and an integral gain.

\subsection{Case 2 (R520S): PI Control with High Force Target}

The force $f_{T}$ in Case 2 was more than double the $f_{T}$ in Case 1, and was selected to be close to the maximum $f_{S}$ determined from the tuning experiments in Section 5. In Case 1, $e_{f}$ was almost always negative due to the low $f_{T}$. The increased $f_{T}$ in Case 2 requires a sign change in $C$ such that $v_{A}>0$ if $e_{f}>0$ since $e_{f}$ will now be primarily positive.

The force $f_{S}$ tends to be low at entry so setting $f_{T}$ close to the maximum $f_{S}$ prevents entry stalling because both $e_{f}$ and $v_{A}$ are large when $f_{S}$ is low (cf. $f_{T}$ and $f_{S}$ in Figures 13 and 15). This setting also makes it much less likely that $f_{S} \rightarrow f_{T}$, which prevents the dig from stalling before the breakout condition is reached. Although less significant, the integral gain also helps increase entry $v_{A}$ since the integral term in $C$ will increase quickly when $e_{f}$ is large.

Unfortunately the higher $f_{T}$ also means that $f_{S}$ can exceed the lifting capacity of the loader. Exceeding the lifting capacity of the loader is mitigated by making the PI controller double sided so that $f_{S}$ is immediately reduced by curling down when $f_{S}>f_{T}$. This situation

should only occur briefly due to the high $f_{T}$, and hence should reduce the risk of dig stalling without increasing the risk of unloading the front axel. 
Table 1: Parameters selected for the PI controller after preliminary tuning tests.

\begin{tabular}{cc}
\hline Parameter & Value \\
\hline$k_{p}$ & $1.0 \times 10^{-7}$ \\
$k_{i}$ & $1.2 \times 10^{-7}$ \\
$f_{T}$ & $178 \mathrm{kN}$ \\
$\delta t$ & $0.099 \mathrm{~s}$ \\
$E_{f_{\max }}$ & $1.25 \times 10^{4} \cdot \delta t \mathrm{~N} \cdot \mathrm{s}$ \\
\hline
\end{tabular}

The double-sided PI controller is given by

$$
v_{A}=k_{p} e_{f}+k_{i} E_{f},
$$

where $k_{i}>0$ is the integral gain, and $E_{f}$ is the trapezoid integral approximation of the error $e_{f}$ defined by

$$
\left(E_{f}\right)_{i}= \begin{cases}\left(E_{f}\right)_{i-1}+\left[\left(e_{f}\right)_{i}+\left(e_{f}\right)_{i-1}\right] \frac{\delta t}{2} & \text { if }-E_{f_{\max }}<\left(E_{f}\right)_{i}<E_{f_{\max }} \\ E_{f_{\max }} & \text { if }\left(E_{f}\right)_{i} \geq E_{f_{\max }} \\ -E_{f_{\max }} & \text { if }\left(E_{f}\right)_{i} \leq-E_{f_{\max }}\end{cases}
$$

where $i$ is the time step index, $\delta t$ is the time in seconds between samples, and $E_{f_{\max }}$ is the maximum value of the trapezoid integral. The integral limit $-E_{f_{\max }}<\left(E_{f}\right)_{i}<E_{f_{\max }}$ serves as an anti-windup condition on the integrator.

Preliminary tuning experiments (Section 5.1.3) were used to select the PI controller parameters. These parameters are listed in Table 1. Both the PI and P parameters were determined experimentally by adjusting them until the payload mass in the bucket was high and consistent. Future work could involve developing off-line tuning methods for $C$ so that the controller gains could be pre-calculated based on known vehicle parameters, and estimated or modelled rock pile stiffness.

\subsection{Case 3 (ST14): Two-Sided Proportional Control}

Following the experiments based on Cases 1 and 2 (above), we chose to deploy a proportionaltype (P) admittance relationship for the full-scale underground experiments on the ST14. The $\mathrm{P}$ admittance controller was selected due to a limited one-week testing schedule in the underground environment, and our desire to focus on the relationship between the admittance controller gain $k_{A}$ and the force target $f_{T}$ over spending time tuning multiple parameters in a PID type controller. In this instance, the admittance relationship is given by

$$
v_{A}=k_{A} e_{f},
$$


where the admittance gain $k_{A}>0$. Descriptions of how changes to the controller gain $k_{A}$ and force threshold $f_{T}$ affect performance are provided in Section 6 .

\subsection{Entry Pose and Thrusting}

For each experiment, the vehicle's drivetrain commands were set such that the machine drives straight into the pile with constant throttle and (in some cases) brake commands. The entry position was selected such that the machine was roughly in line with the centre of the muck pile. This alignment was set by eye and was only necessary to ensure relatively consistent confinement provided by the rock surrounding the entry point.

The entry pose was set such that the bucket scraped the ground to ensure the bucket penetrates the bottom of the rock pile. For experiments with the Kubota R520S, the throttle was set to full to maximize the bucket actuator speed and power while the forward thrust was limited by applying partial brake. The brake level was set such that the forward thrust limited $f_{S}$ to within the controllable range of the actuators. For experiments with the Atlas Copco ST14 LHD, full throttle was used, in first gear, and with no brake.

\section{FIELD TEST APPARATUSES AND METHODS}

Two experimental vehicles and two distinct environments were used to field test the Autonomous Loading Controller (ALC): 1) a smaller 1-tonne wheel loader in an outdoor environment at Innovation Park, Queen's University; and, 2) a full-scale 14-tonne LHD in an underground mine at Kvarntorp, Sweden. The field tests at Queen's were used to test and tune the Case 1 and Case 2 loading algorithm and admittance controllers discussed in Section 3.1. The experience gained from these tests was used to prepare for full scale testing of the Case 3 loading algorithm on the ST14 in Sweden.

\subsection{Environments}

The R520S field tests at Queen's were conducted outdoors with a blasted limestone muck pile resting on an asphalt surface. While several weather conditions were experienced, the effect of these conditions was not distinguishable in the tests results. The tests presented in this paper were conducted over a four day period with similar weather conditions.

The ST14 field tests at Kvarntorp were conducted in a decommissioned underground limestone room-and-pillar mine with blasted limestone and granite muck piles. The test area was located approximately $30 \mathrm{~m}$ below surface, where the tunnels (called mine drifts) are approximately 10-12 $\mathrm{m}$ wide and $6 \mathrm{~m}$ tall. The roadway was gravel and clay over the limestone drift floor. Both environments are shown in Figure 6. A map of the underground environment showing the limestone muck pile along the wall of Drift 159, and the granite muck pile at the end of Drift 165 is shown in Figure 7. 


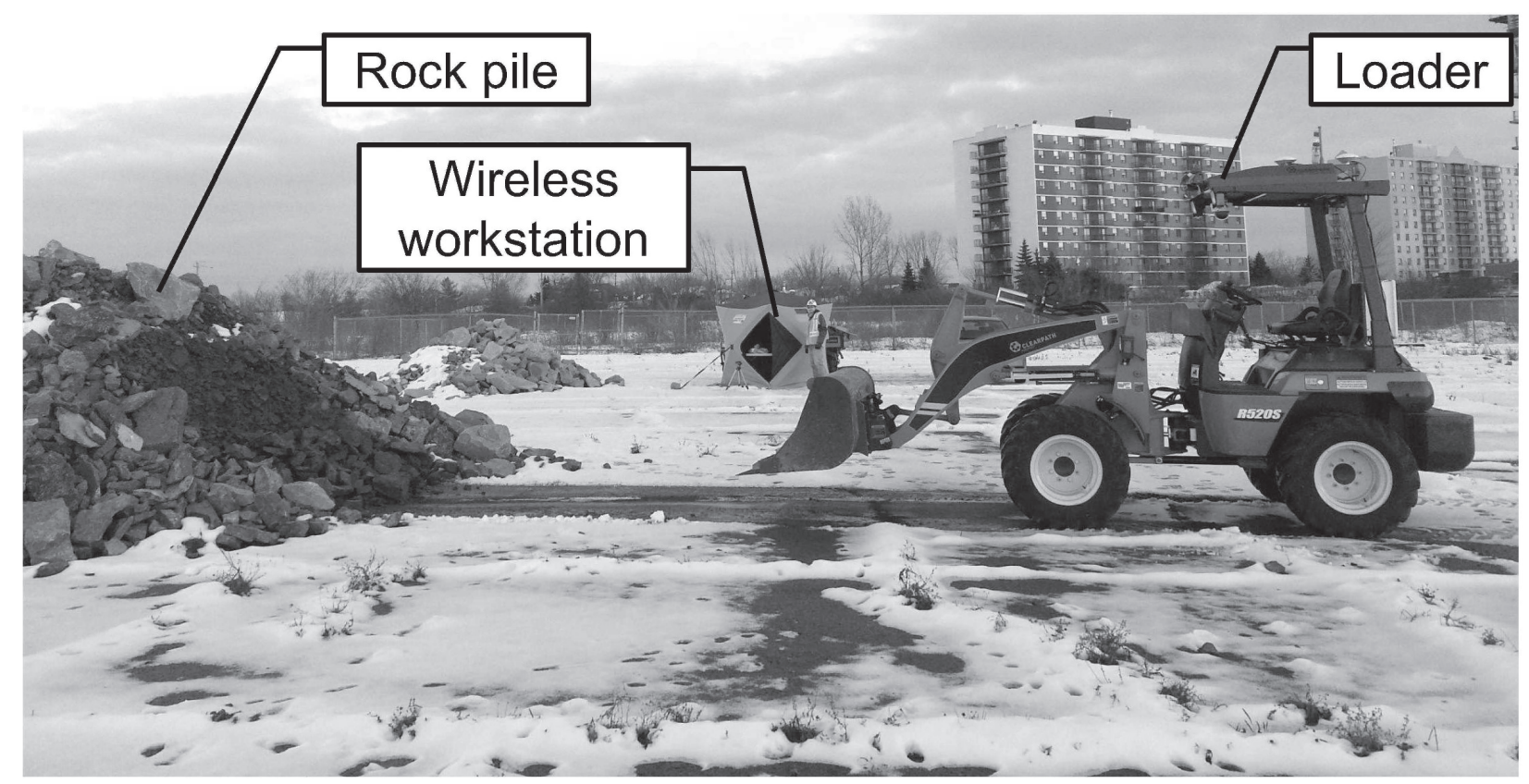

(a) R520S surface test environment at Queen's

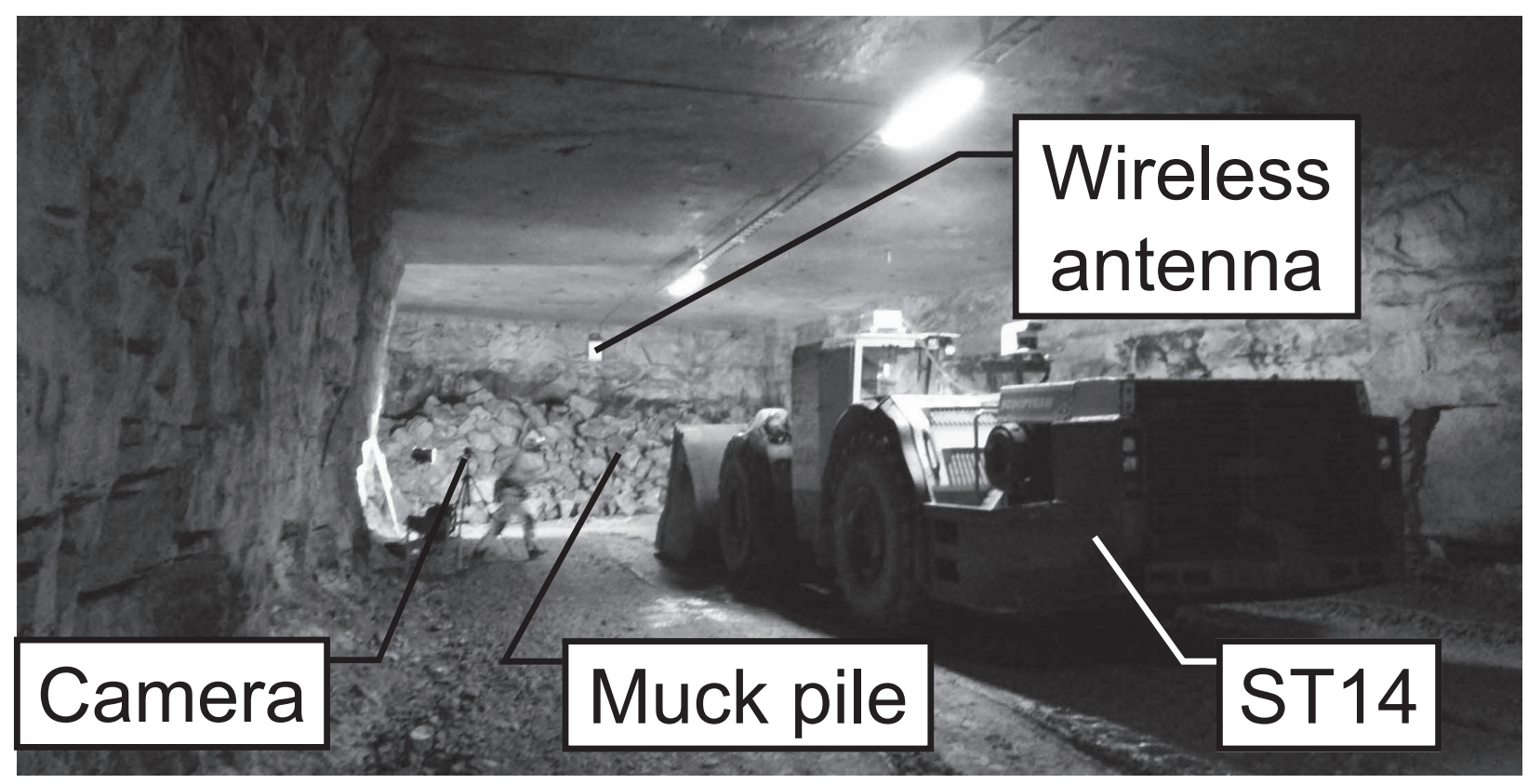

(b) ST14 underground test environment at Kvarntorp

Figure 6: The R520S loader tests were performed outdoors on an asphalt surface (a), while the ST14 tests were conducted in an underground mine on a limestone roadway covered with approximately $30 \mathrm{~cm}$ of gravel and clay (b). Both vehicles were positioned in front of their respective muck piles before each dig attempt. Video cameras located on to the side of the vehicles and muck piles were used to provide context for each dig attempt. 


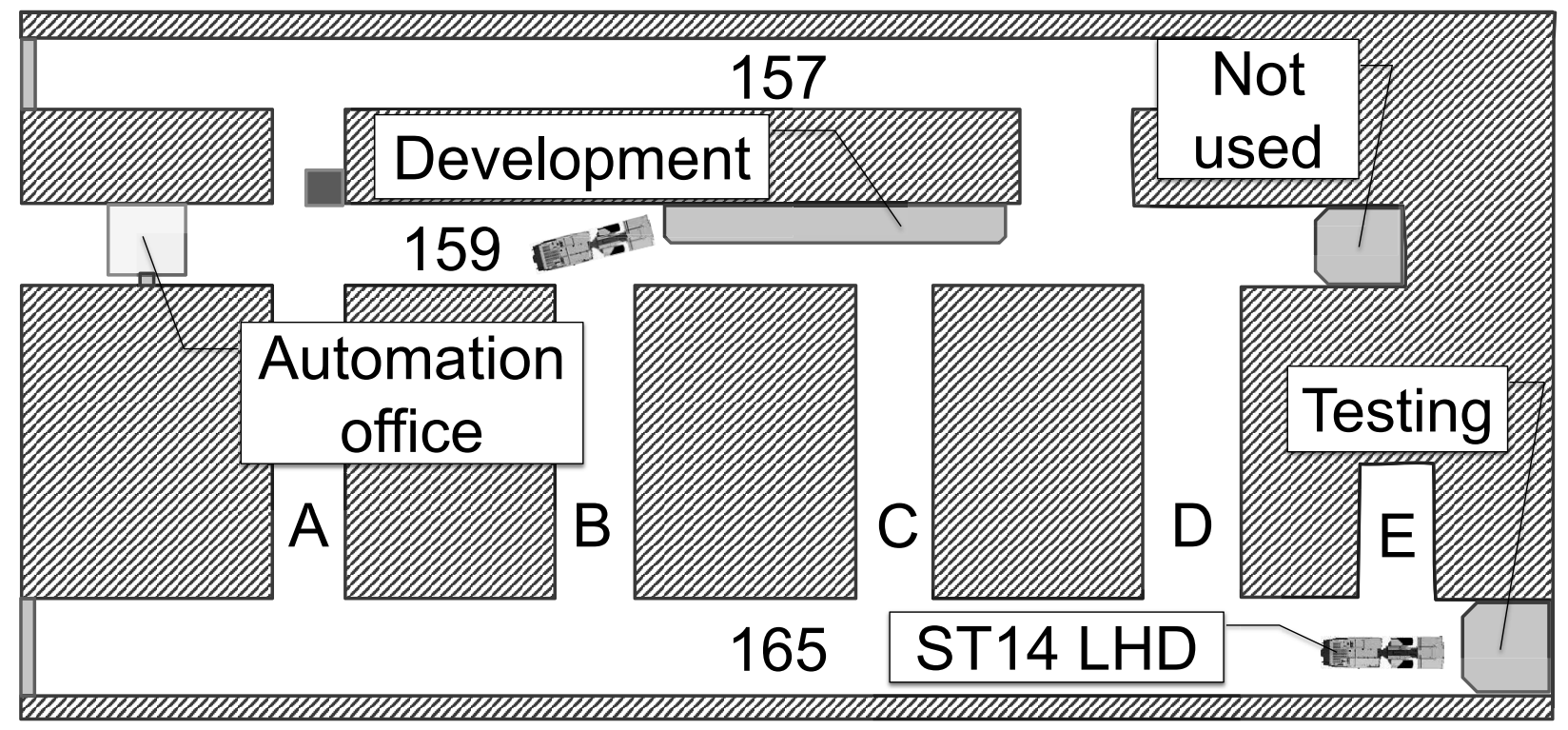

Figure 7: The limestone muck pile along the wall of Drift 159 was composed of finer limestone fragments compared to the granite muck pile at the end of Drift 165. Preliminary tuning tests were conducted in the limestone muck pile while both the manual and autonomous loading tests were conducted in the granite muck pile.

\subsection{Muck Piles}

The limestone muck pile at Queen's is composed of blasted local limestone with a mean $( \pm 1 \sigma)$ density of $2.65 \pm 1.12 \times 10^{3} \mathrm{~kg} / \mathrm{m}^{3}$, and a mean rock size of $0.063 \pm 0.039 \mathrm{~m}$. Two muck piles were used for the tests in Kvarntorp. The first pile (Drift 159) is composed of local fragmented limestone while the second pile (Drift 165) is composed of over $200 \mathrm{t}$ of imported blasted granite. The two piles had similar densities of $2.72 \pm 0.60 \times 10^{3} \mathrm{~kg} / \mathrm{m}^{3}$, and $2.47 \pm 1.28 \times 10^{3} \mathrm{~kg} / \mathrm{m}^{3}$ respectively, but dissimilar mean rock sizes of $0.20 \pm 0.09 \mathrm{~m}$, and $0.48 \pm 0.19 \mathrm{~m}$ respectively. These three muck piles are shown in Figure 8 . The density measurements were obtained by using water displacement experiments, and the size distributions were obtained by using visual fragmentation measurement techniques. The largest visible rock size dimensions were roughly estimated by using a Gaussian edge detection algorithm (as implemented in the GNU image manipulation program GIMP version 2.8) on a $0.75 \mathrm{~m}$ by $0.75 \mathrm{~m}$ region for the MSL muck pile, and a 2 by $3 \mathrm{~m}$ region of the muck piles in Kvarntorp. This manual method is biased towards fragments greater than $0.013 \mathrm{~m}$ and is error prone, but is sufficient for illustrating the wide size distribution relative to the gravel and soil experiments conducted by other researchers.

There were also much larger fragments outside of the measurement regions, such as the $0.52 \mathrm{~m}$ rock that was used to scale the fragments in Figure 8(a). These larger fragments may have also been hidden below the muck pile surface, which highlights the difficulties in modelling muck piles compared with soils or gravel. Figure 8(a) also illustrates the tendency for fine material to migrate towards the centre of the muck pile, leaving larger material on 


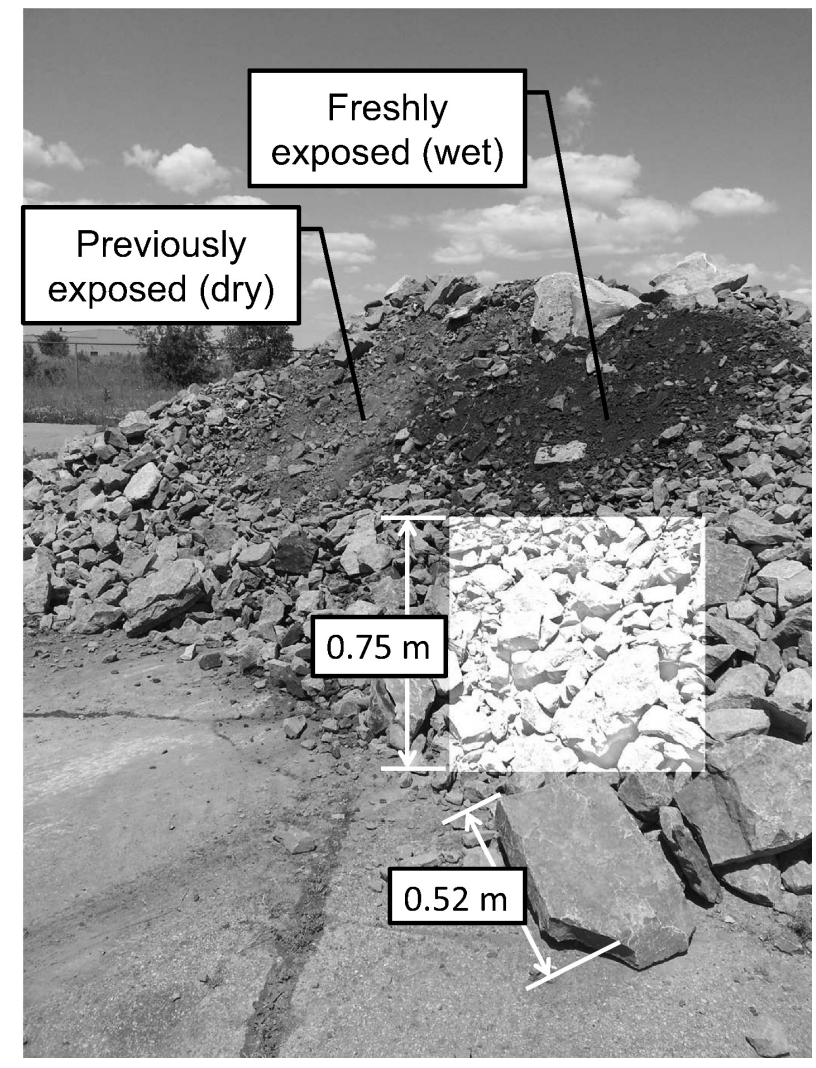

(a) MSL muck pile

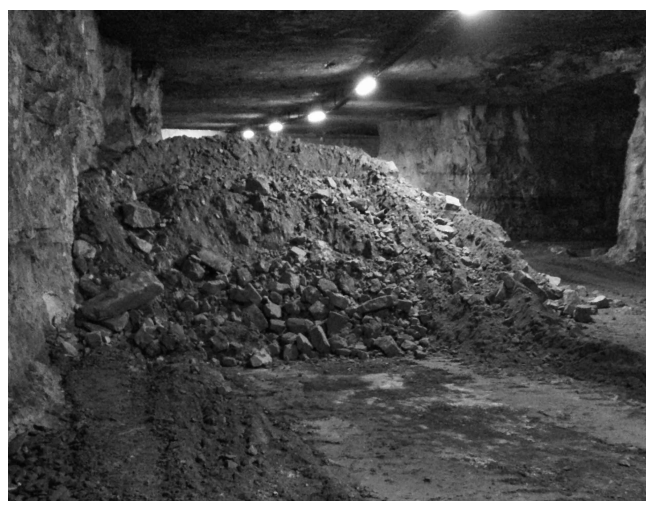

(b) Drift 159 muck pile

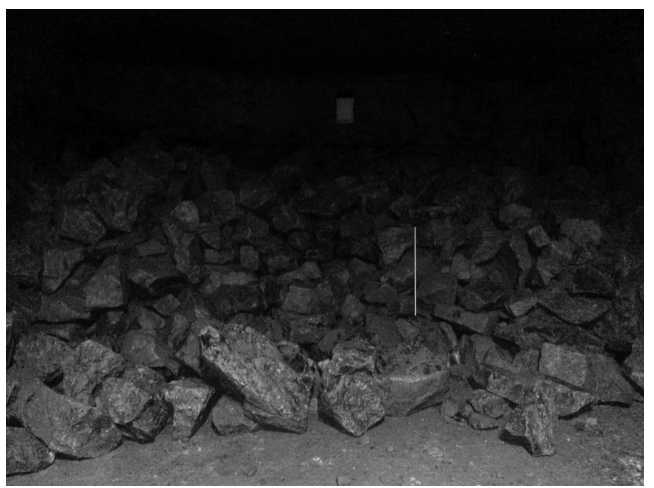

(c) Drift 165 muck pile

Figure 8: The $0.52 \mathrm{~m}$ rock in the foreground of the MSL muck pile (a) was used to provide scale to the rock fragments within the highlighted $0.75 \mathrm{~m}$ box. A Gaussian edge detection algorithm was used to estimate the rock edges in the $0.75 \mathrm{~m}$ box. The limestone muck pile in Drift 159 (b) was used for preliminary tests and tuning, while manual tests, final tuning, and ALC evaluation tests were conducted in the granite muck pile in Drift 165 (c). 
the exterior. The freshly exposed material is appreciably darker than the previously exposed material indicating a higher moisture content. Both the fines and the moisture content tend to increase pile cohesion and stiffness, which makes digging deeper into the pile more difficult.

\subsection{Loading Machines}

Figure 6 shows both the 1-tonne (bucket capacity) Kubota R520S wheel loader used for the tests conducted at Queen's in Canada, and the 14-tonne (bucket capacity) Atlas Copco Scooptram ST14 used for the tests conducted at the Kvarntorp mine in Sweden. Both vehicles are hydraulically-actuated and centre-articulated. Furthermore, both vehicles use the same hoist and curl mechanism, shown in Figure 4.

\subsubsection{Modified Kubota R520S}

The Kubota R520S loader was retrofitted for automation by Clearpath Robotics (Kitchener, $\mathrm{ON})$. Although the loader has several sensors, only the boom and bucket extension and pressure sensors were used for the experiments presented here. Figure 9 shows the arrangement of the custom modifications. The boom and bucket actuator extensions were measured at $10 \mathrm{~Hz}$ by a custom hall effect sensor. Each extension sensor contains two Honeywell SPSL225 contactless IP69 linear encoders mounted in a custom housing. Two Measurement Specialties MSP-400 pressure sensors were installed on the rod and actuator ports of each actuator so that the net force acting on the actuators can be calculated. The pressure sensor data was captured at $107 \mathrm{~Hz}$ by a single Arduino Uno. The different sampling rates were due to the different data acquisition methods between the Clearpath system and the Arduino Uno. The higher sampling rate for the pressures allowed for a more detailed study of the noisy digging forces. A lower sampling rate (due to inefficiencies in the Arduino code) of $87 \mathrm{~Hz}$ was also used for some digs, but controller performance was not affected.

The Arduino pressure and actuator extension messages were passed to the main computer over a ROS Electric network. The main computer was a Mini-ITX Intel Core i5 64-bit PC running Ubuntu 11.10, and ROS Electric. The main computer used a ROS network to publish and subscribe to topics over a wireless network. The ALC was run on a separate Intel Core i5 64-bit laptop (running Ubuntu 11.10, and ROS Electric) connected to the wireless network. This laptop was also used for data collection.

The Kubota R520S uses a fixed displacement hydraulic pump and open-centre hydraulic valves to control the hydraulic flow to all of the actuators. The fixed displacement pump means that the supply pressure to the actuators depends on both the engine throttle, and the number of actuators being used. While full throttle is best for maximizing the applied force of the actuators, it also means that the drive train will create very high thrust loads when in contact with the muck pile. These high thrust loads can exceed the lifting capacity of the actuators causing dig attempts to stall, and sometimes tire slip. This deficiency was partially mitigated by using the brake to limit forward motion (see also Section 3.5). 


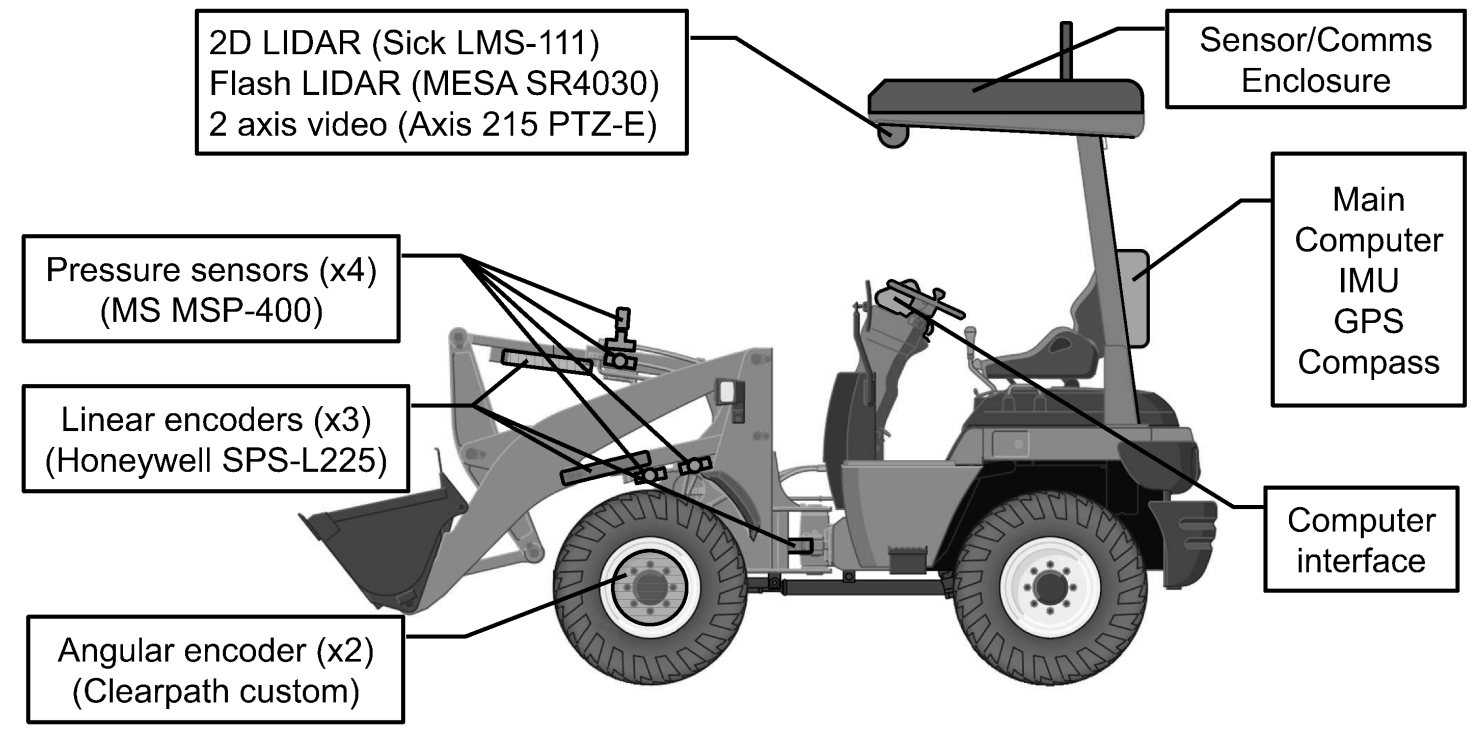

Figure 9: Clearpath Robotics added several sensors and systems to automate the stock Kubota 520s. Only the pressure sensors, linear encoders, driving system, main computer, and communications systems are used by the loading algorithm presented in this paper.

Clearpath replaced the original Kubota linkages between the manual control levers and the boom and bucket control valves with rotary servos driving an armature. These servos had poor repeatability, especially after reaching steady state temperature. This repeatability problem caused the boom and bucket to deviate from the intended entry pose and led to dig failures due to front axle unloading. The three position switches used to control these servos on board the vehicle also lacked the resolution necessary to obtain manual digging data. Both the repeatability of the servos, and the fixed displacement pump also resulted in significant non-linear system response. These non-linearities made the loader (and process) difficult to model, such that off-line admittance controller analysis was abandoned in favour of manual tuning in the field.

\subsubsection{Atlas Copco ST14 ARV}

The Atlas Copco Scooptram ST14 pictured in Figure 10 has a Cummins SM11 EPA Tier 3/EU Stage IIIA diesel engine. This engine provides a maximum torque of $1674 \mathrm{Nm}$ at $1350 \mathrm{rpm}$. It has four driving gear positions. The bucket can hold $14 \mathrm{t}$ and has a nominally heaped volume of $6.4 \mathrm{~m}^{3}$. The nominal dimensions of the vehicle (including the bucket) are $10.825 \mathrm{~m}$ long, $2.550 \mathrm{~m}$ tall, and $2.800 \mathrm{~m}$ wide. The vehicle is also equipped with a Rig Control System (RCS), which is Atlas Copco's custom on-board computerized control architecture (Atlas Copco, 2012). The ST14 model used for these tests was an Automation Ready Vehicle (ARV), which includes one forward and one rear facing SICK LMS LiDAR, several video cameras located around the vehicle, wheel encoders, steering and boom angle encoders, a bucket actuator linear sensor, actuator pressure sensors, an inertial measurement unit, data acquisition and communications systems, and an onboard computer system. The primary 


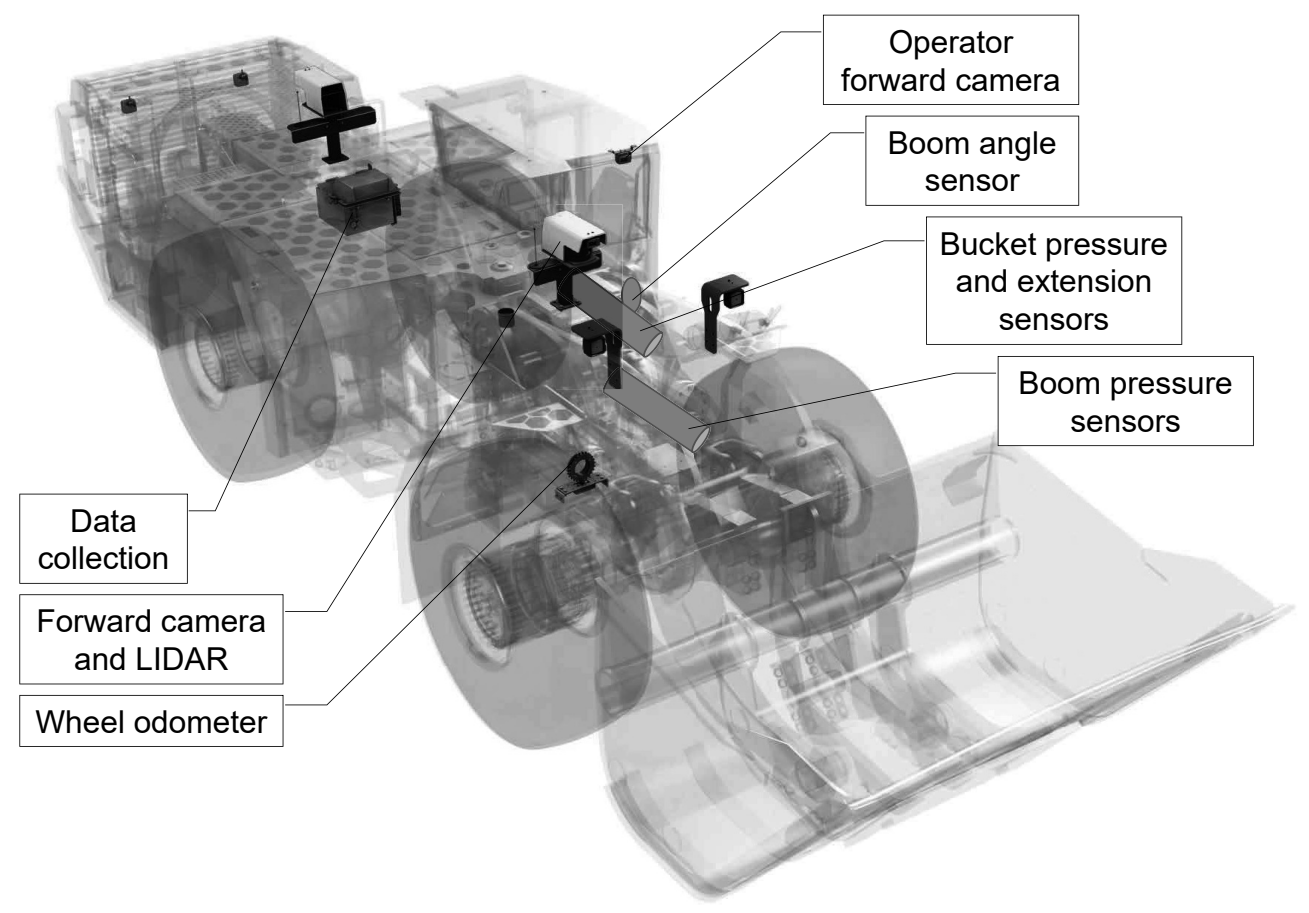

Figure 10: The Atlas Copco SCooptram ST14 has a bucket capacity of $14 \mathrm{t}$. Its actuators are driven by a close-center, constant pressure hydraulic system, and it can be controlled remotely by using an Atlas Copco Rig Control System. The primary sensors used for these field tests were the pressure and linear sensors mounted on the curl and hoist actuators (Reproduced and altered with permission from Atlas Copco).

sensors used by the ALC were the boom angle sensor, the linear bucket actuator sensor, and the pressure transducers for both boom and bucket actuators.

In contrast to the R520S, the ST14 has a closed-centre, constant-pressure hydraulic system. In a closed centre hydraulic system, the fluid cannot return to the hydraulic supply when the control valves are closed. The hydraulic system maintains constant pressure by bypassing a portion of the supplied fluid through a compensating valve that alters the swash plate angle in the variable displacement pump.

\subsection{ALC Test Procedures}

The test setup for each dig attempt is shown for both environments and platforms in Figure 6. The preliminary R520S tuning experiments were conducted first, followed by digging with a fully saturated controller (i.e., simply curl the bucket at full speed upon penetration), and then with the controllers described in Sections 3.2 and 3.3. Comparing the saturated case with the controlled cases helps to illustrate the difference between curling at maximum velocity and using the admittance controller to match curl velocity to the reaction forces. Once the loading algorithm was tuned for this loader and muck pile, the various loading algorithm parameters were held constant for the dig attempts plotted in Figure 16 . The 


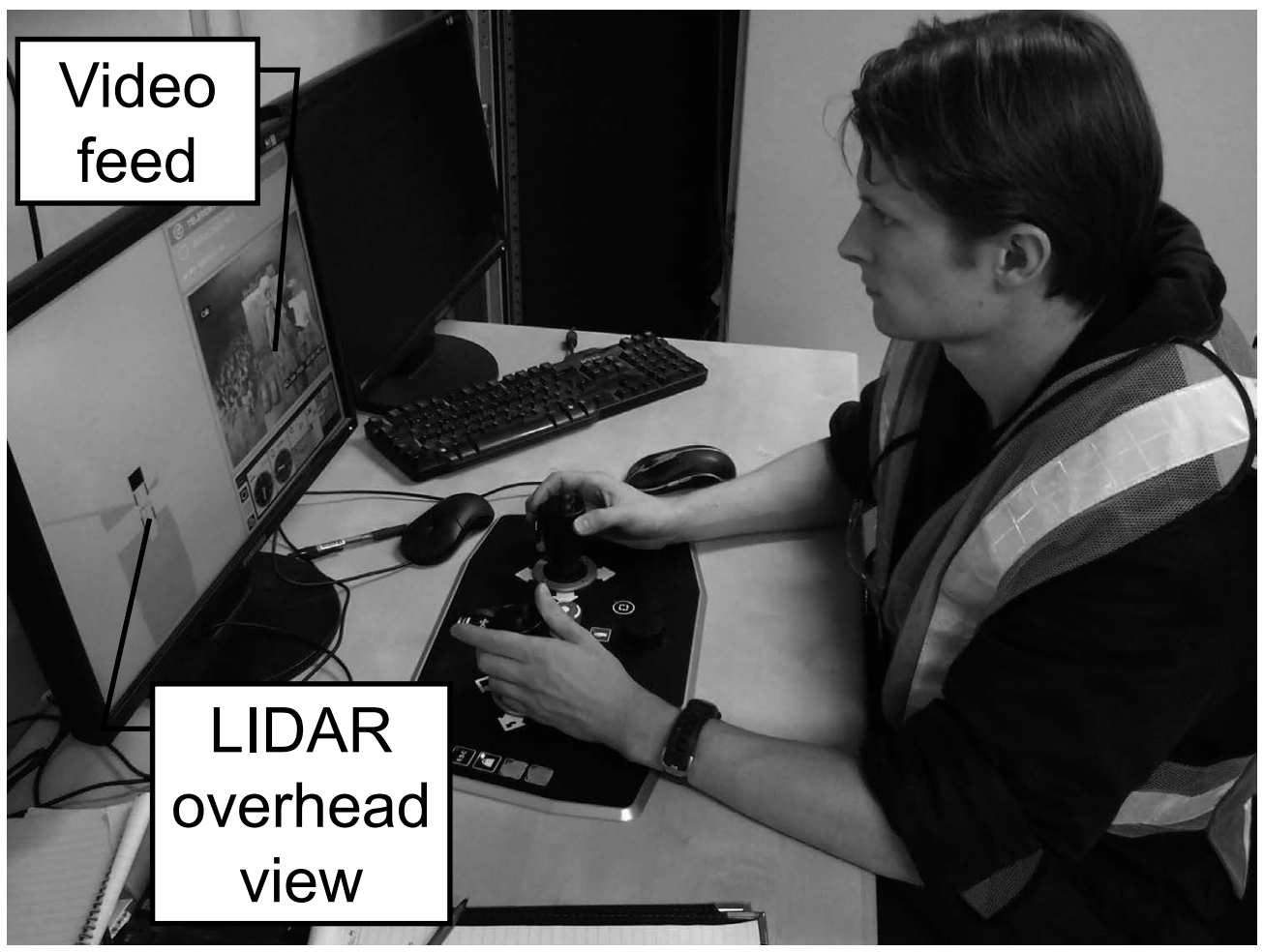

Figure 11: The automation office contained an operator station (OPS) for teleremote control of the ST14.

R520S field tests were followed by the full scale ST14 tests conducted in the Kvarntorp Mine by using the controller described in Sections 3.4.

For all tests (both vehicles), each dig attempt started by manually moving the loader in front of the muck pile. The loading algorithm then assumed control of the vehicle for entry, digging, breakout, and weighing. A weight reading was taken, and the volume of rock within the bucket was verified by using the video feeds taken from cameras located near the muck piles. The payload was then dumped manually, and the vehicle was re-positioned in front of the muck pile.

While this procedure was followed for all tests, each environment and platform had slightly different requirements. The ST14 test days began by warming up the vehicle for 10 to $20 \mathrm{~min}$ before the ST14 was positioned approximately $11 \mathrm{~m}$ back from the muck pile toe. The ST14 was completely unmanned and was teleoperated from an operator station, as shown in Figure 11, located at the entrance of Drift 159. The R520S required a rider to hold down a deadman's switch while a second operator ran the ALC program from a wireless workstation adjacent to the muck pile. All tuning tests in Kvarntorp were conducted by using the muck pile along the wall of Drift 159, while the manual and ALC comparison tests were conducted in the granite muck pile at the end of Drift 165. 


\subsection{ST14 Manual and ALC Comparison Test Procedure}

For the manual dig trials, our expert operator "Frank" was instructed to: 1) dig normally by using both boom and bucket actuators; 2) dig in a manor similar to the ALC by using only the bucket actuator; and 3) dig at $50 \%$ throttle. The $50 \%$ throttle setting was selected to determine whether there were any advantages to digging at lower throttle. The manual dig efficiency results shown in Figure 25 indicate that digging at lower throttle should be avoided and hence the ALC throttle setting was set to $100 \%$ to better match the bucket only and both actuator manual digging methods. Similarly, the entry velocity was also selected to match the manual dig attempts and averaged $5.0 \mathrm{~km} / \mathrm{hr}$. This velocity corresponds to $100 \%$ throttle, first gear, and $0 \%$ brake.

In all tests, Frank controlled the vehicle from inside the ST14. The ALC digs began by switching the ST14 to "automation mode". The operator then left the vehicle, and entered the automation office shown in Figure 11. After uploading the desired tuning parameters to the ST14, the ALC was initiated. When the ALC reached its final state, the ST14 was switched to teleremote mode so that the bucket could be lifted, weighed, and dumped. The same weighing and dumping procedure was performed by Frank following his dig attempts.

\section{KUBOTA R520S FIELD EXPERIMENT RESULTS}

Field experiments involved both tuning and evaluating several digs by using the tuned parameters. The flowcharts in Figure 12 illustrate the approaches taken to tune the brake setting, dig target force, proportional gain, and integral gain sequentially. The R520S tuning results indicate the importance of the brake setting, the admittance gain, and the dig target $f_{T}$ on loading.

\subsection{Tuning Experiments}

A new, heavier bucket and the fragmented muck pile pose a significant challenge for the 1-tonne Kubota loader, which was originally designed for soil excavation. The constant displacement pump on the loader means that maximum loader throttle must be used to ensure the actuators can apply full force to the rock. Unfortunately, full throttle means the drivetrain will also thrust into the pile with maximum force, which dramatically increases the digging forces $f_{S}$. If $f_{S}$ exceeds the lifting capacity of the actuators the bucket will stall and the dig will fail. Hence, the first step was to determine a brake setting that would limit the thrust against the pile such that the reaction forces could be overcome by the actuators. It was determined that at $50 \%$ brake the brake level is sufficient to limit the maximum reaction force below $100 \mathrm{kN}$, but not so high that contact is lost with the pile. 


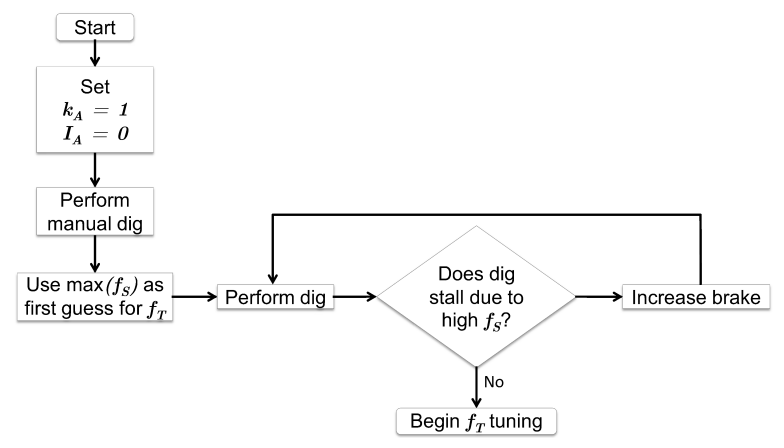

(a) Brake tuning

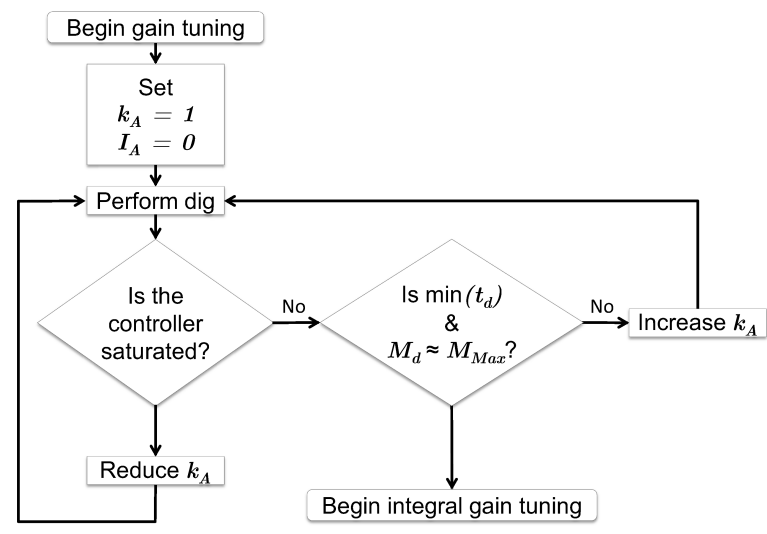

(c) Proportional gain tuning

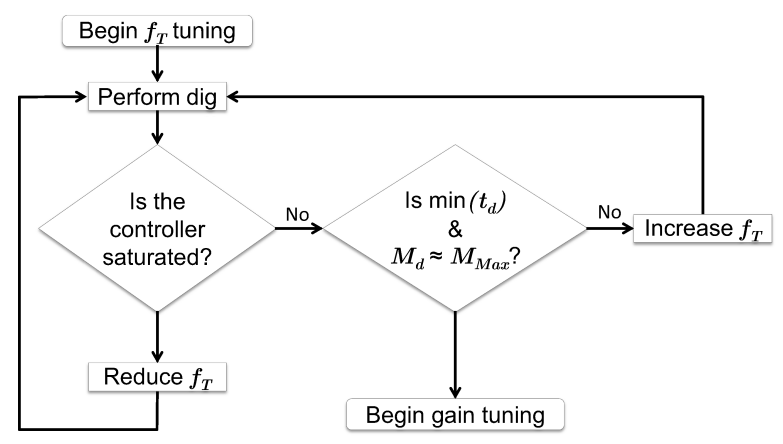

(b) Dig target force tuning

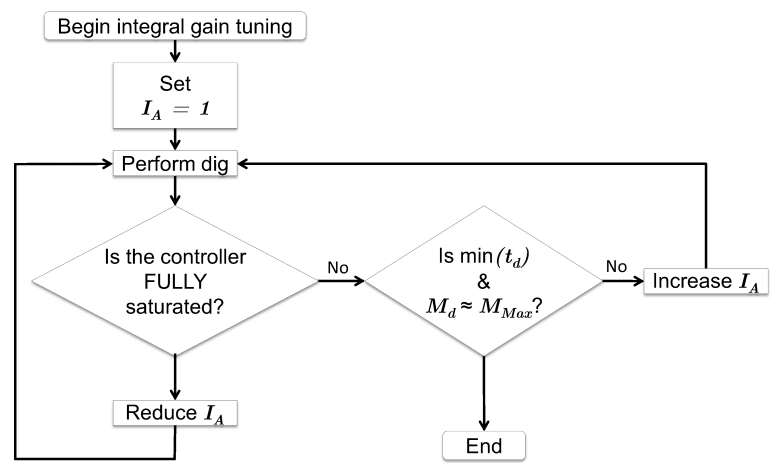

(d) Integral gain tuning

Figure 12: The controllers were tuned until the general performance criteria were met $\left(t_{d}\right.$ minimized while $\left.M_{d} \rightarrow M_{\max }\right)$. 

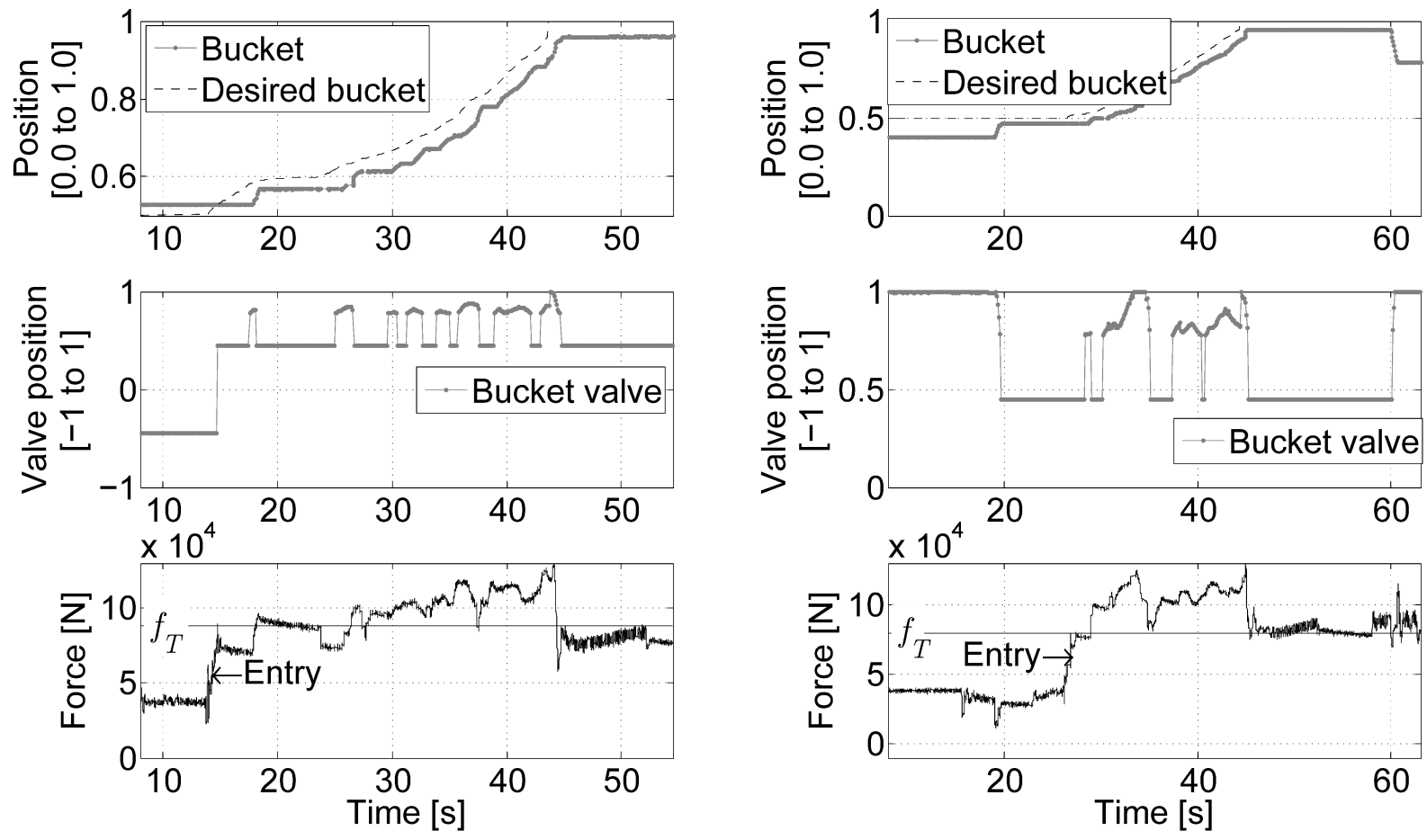

(a) $88 \mathrm{kN}$ dig target

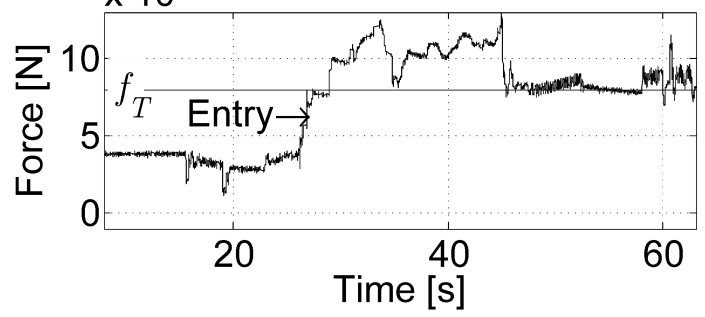

(b) $80 \mathrm{kN}$ dig target

Figure 13: The bucket actuator and valve positions are used along with the boom and bucket forces to show the effect of lowering the dig target force $f_{T}$ from $88 \mathrm{kN}$ to $80 \mathrm{kN}$. While the valve response is excellent in (a) it is still below the maximum valve limit until $42 \mathrm{~s}$ into the dig. $f_{T}$ is decreased from 88 to $80 \mathrm{kN}$ in (b), which increases valve saturation because the force error between $f_{T}$, and $f_{S}$ increases.

\subsubsection{Case 1: Target Force Tuning}

The goal of tuning $f_{T}$ is to minimize dig time while reliably obtaining payloads close to the maximum payload of the loader. This goal was achieved by using the tuning process depicted in Figure 12(b). Figure 13 shows the dig response when $f_{T}$ is dropped from 88 to $80 \mathrm{kN}$. The boom and bucket extensions are normalized between 0 and 1 , while the valve positions are normalized between -1 and 1 . The valve plots from $f_{T}=88 \mathrm{kN}$ only reached 1.0 once near the end of the dig when the error $e_{f}$ between $f_{T}$ and the sensed forces $f_{S}$ reached its maximum. Dropping $f_{T}$ to $80 \mathrm{kN}$ allowed the admittance controller to issue slightly higher desired positions earlier without completely saturating the admittance controller.

\subsubsection{Case 1: P Gain Tuning}

The goal of tuning the proportional gain is to minimize dig time while reliably obtaining payloads close to the maximum payload of the loader. This goal is achieved by using the tuning process depicted in Figure 12(c). Figure 14 shows the dig response when the $\mathrm{P}$ controller admittance gain $k_{p}$ is dropped from $10 \times 10^{-6}$ to $1 \times 10^{-6}$ with the brake held 
constant at $50 \%$, and $f_{T}=80 \mathrm{kN}$. At $10 \times 10^{-6}$ both the change in desired bucket position (curl velocity), and the valve positions are at their maximum values throughout the dig. In other words, the admittance controller is completely saturated. The admittance controller is less saturated at $k_{p}=5 \times 10^{-6}$, and never saturated at $k_{p}=1 \times 10^{-6}$. The valve response when $k_{p}=1 \times 10^{-6}$ showed excellent range between the deadband at 0.50 , and just below 1.00 , which is the maximum valve position.

\subsubsection{Case 2: PI Admittance Controller Tuning}

The PI admittance controller used the same tuning approach as the $\mathrm{P}$ admittance controller except for a higher initial $f_{T}$ such that $f_{S}$ tended to be less than $f_{T}$, and an initial integral gain $k_{i}$ of $1.0 \times 10^{-7}$. At $k_{i}=1.0 \times 10^{-7}$, tuning experiments similar to the ones discussed in Case 1 resulted in $k_{p}=1.0 \times 10^{-7}$ and $f_{T}=178 \mathrm{kN}$ to minimize dig time while increasing payload towards the maximum rated payload of the loader. The specific tuning process for $k_{i}$ is shown in Figure 12(d). Then $k_{i}$ was increased from $1.0 \times 10^{-7}$ to $1.2 \times 10^{-7}$ based on the response plots in Figure 15. Increasing $k_{i}$ tended to increase the valve response during the dig when $e_{f}$ began to stagnate, but was not approaching 0 .

These plots show that the PI controller logic of Case 2 increases $e_{f}$ at the beginning and end of each dig when increased bucket velocity is most desirable to prevent stalling. Controller saturation is also reduced between Figures $15(\mathrm{a})$ and $15(\mathrm{c})$, which indicates that $k_{p}$, and $k_{i}$ were adequately matched to this loader and muck pile. Once the PI controller was tuned its parameters were left constant for the final dig trial.

\subsection{Defining Dig Efficiency}

In order to evaluate the effectiveness of the robotic loading algorithm, we define dig efficiency $\epsilon_{d}$ as a combination of three metrics: 1) the dig time $\left.t_{d}, 2\right)$ the actuator work expended while digging $W_{d}$, and 3 ) the mass of rock in the bucket at the end of the dig attempt $M_{d}$ (also called the payload). Together these three metrics define an efficiency point $\epsilon_{d}\left(t_{d}, W_{d}, M_{d}\right)$.

Figure 16 shows the dig efficiency points for 57 robotic loading attempts. Digs with high dig efficiency appear near to the 0 work, 0 time axis, while achieving high payloads. It is also desirable that the dig efficiency results are consistent so that mine operators can decrease scheduling and throughput variability.

The payload mass $M_{d}$ was calculated by using the boom force $F_{h}$ and by

$$
M_{d}=\frac{F_{h}-3.9 \times 10^{4}}{51.8} \mathrm{~kg}
$$

which was measured while the loader was in the weighing pose. Equation (12) was determined experimentally by calibrating the loader in the weighing pose by using known masses. 

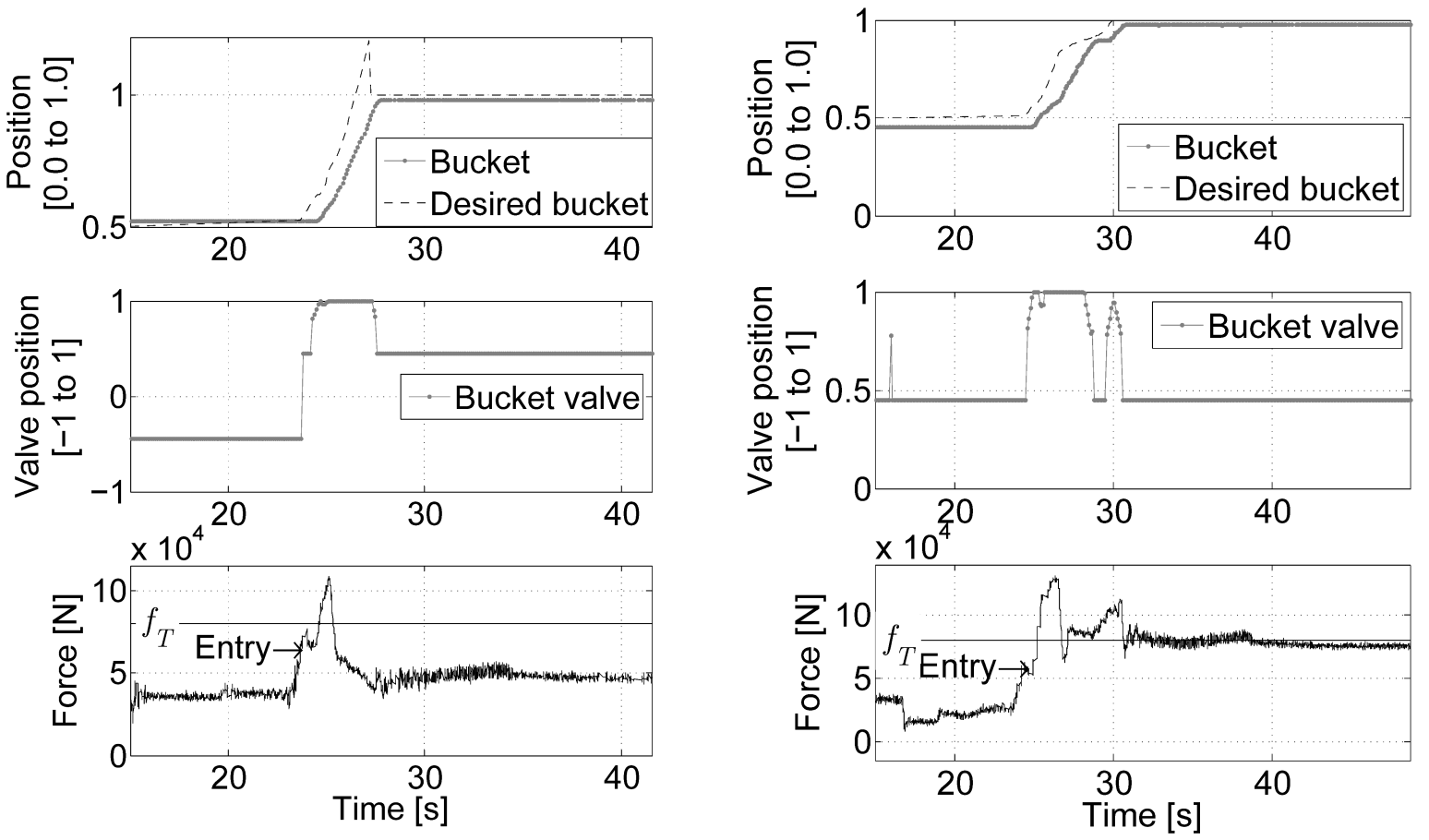

(a) Gain $10 \times 10^{-6}$

(b) Gain $5 \times 10^{-6}$
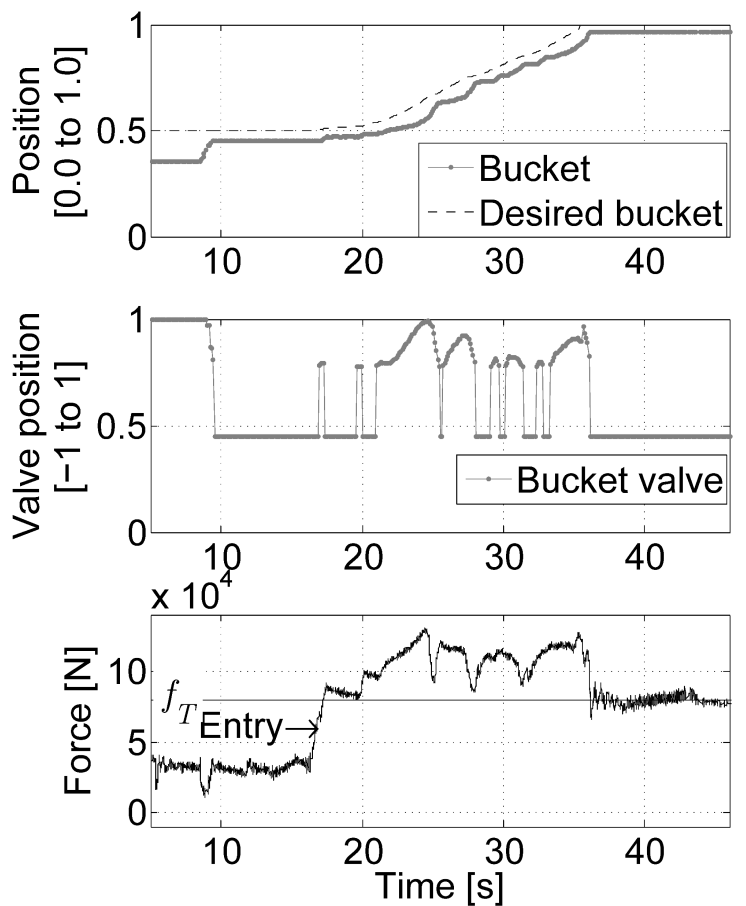

(c) Gain $1 \times 10^{-6}$

Figure 14: The bucket actuator and valve positions are used along with the boom and bucket forces to show the effect of lowering the admittance gain from $10 \times 10^{-6}$ to $1 \times 10^{-6}$. The admittance controller is saturated at a gain of $10 \times 10^{-6}$ (a), mostly saturated at a gain of $5 \times 10^{-6}(\mathrm{~b})$, and has maximum response at a gain of $1 \times 10^{-6}(\mathrm{c})$. 

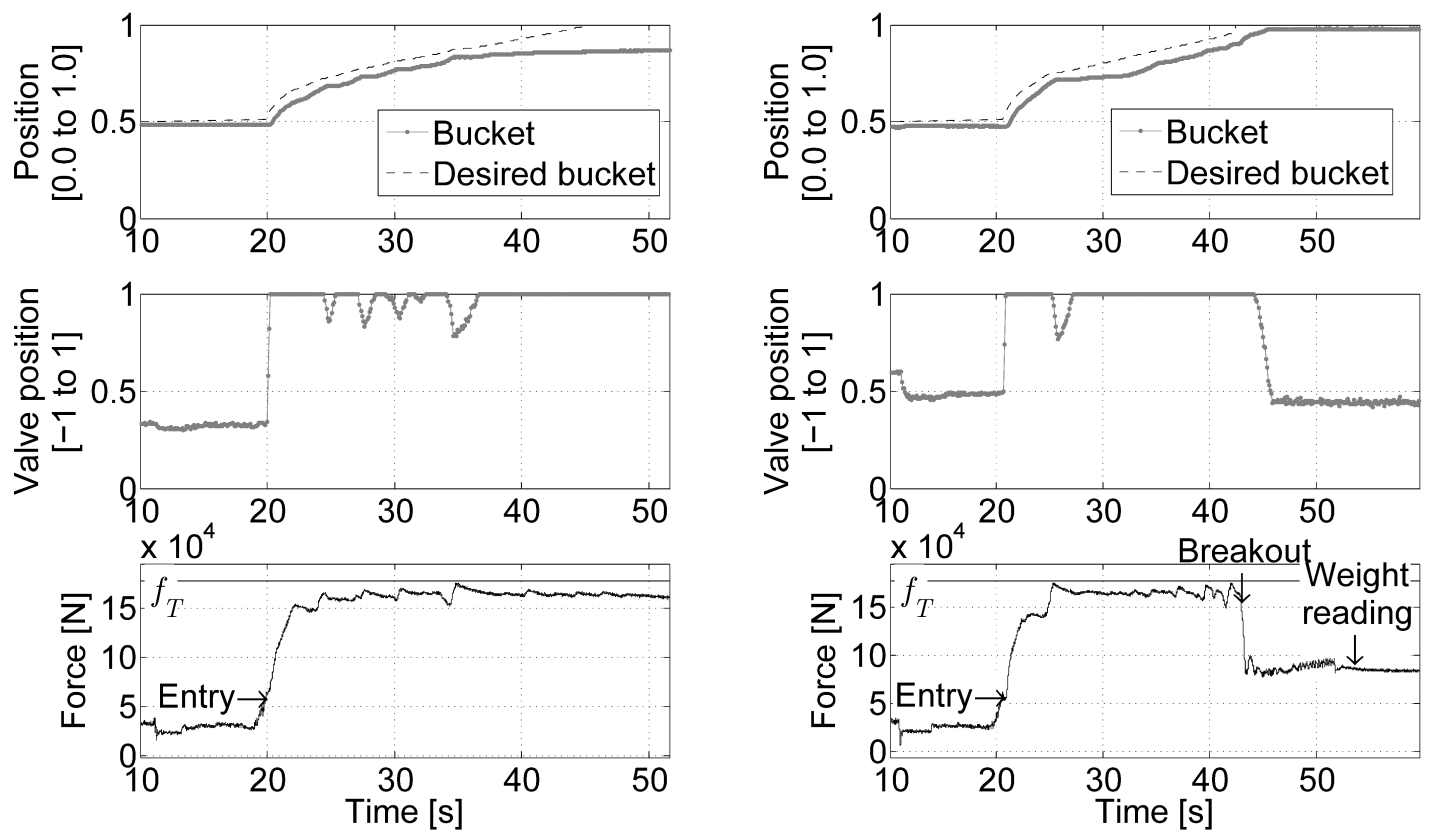

(a) Gain $1.00 \times 10^{-7}$

(b) Gain $1.15 \times 10^{-7}$
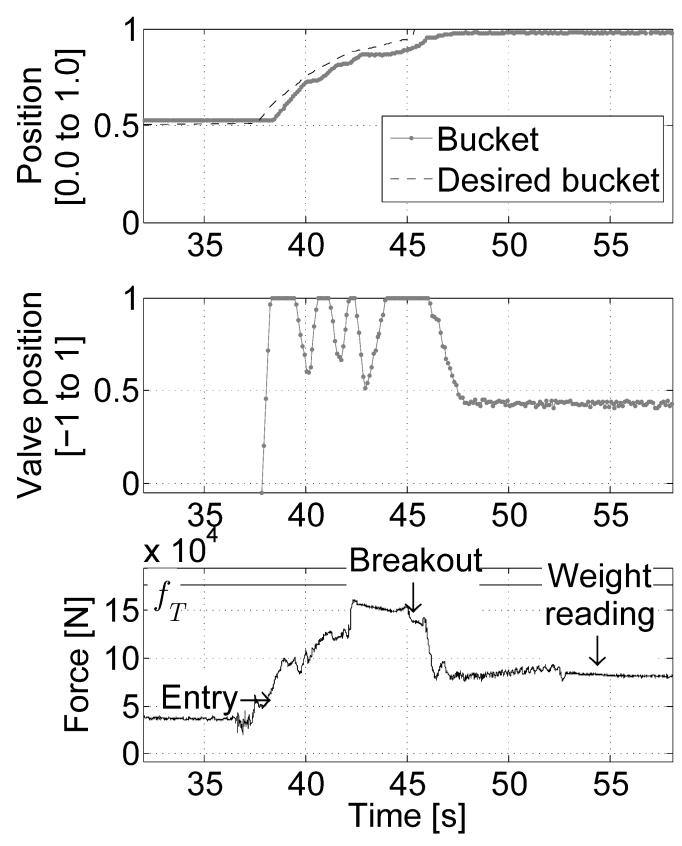

(c) Gain $1.20 \times 10^{-7}$

Figure 15: The bucket actuator and valve positions are used along with the boom and bucket forces to show the effect of increasing the admittance integral gain $k_{i}$ from $1.00 \times 10^{-7}$ to $1.20 \times 10^{-7}$. In (a) $k_{i}$ is set too low resulting in forces that are too close to the dig target $f_{T}$, and the admittance controller stalls. At $1.15 \times 10^{-7}$ (b) $k_{i}$ is high enough that the force drop to $150 \mathrm{kN}$ at $42 \mathrm{~s}$ causes the admittance controller to issue the final curl command. Unfortunately, the controller is mostly saturated. In (c) $k_{i}$ is high enough that the forces remain below the $f_{T}$, without saturating. 


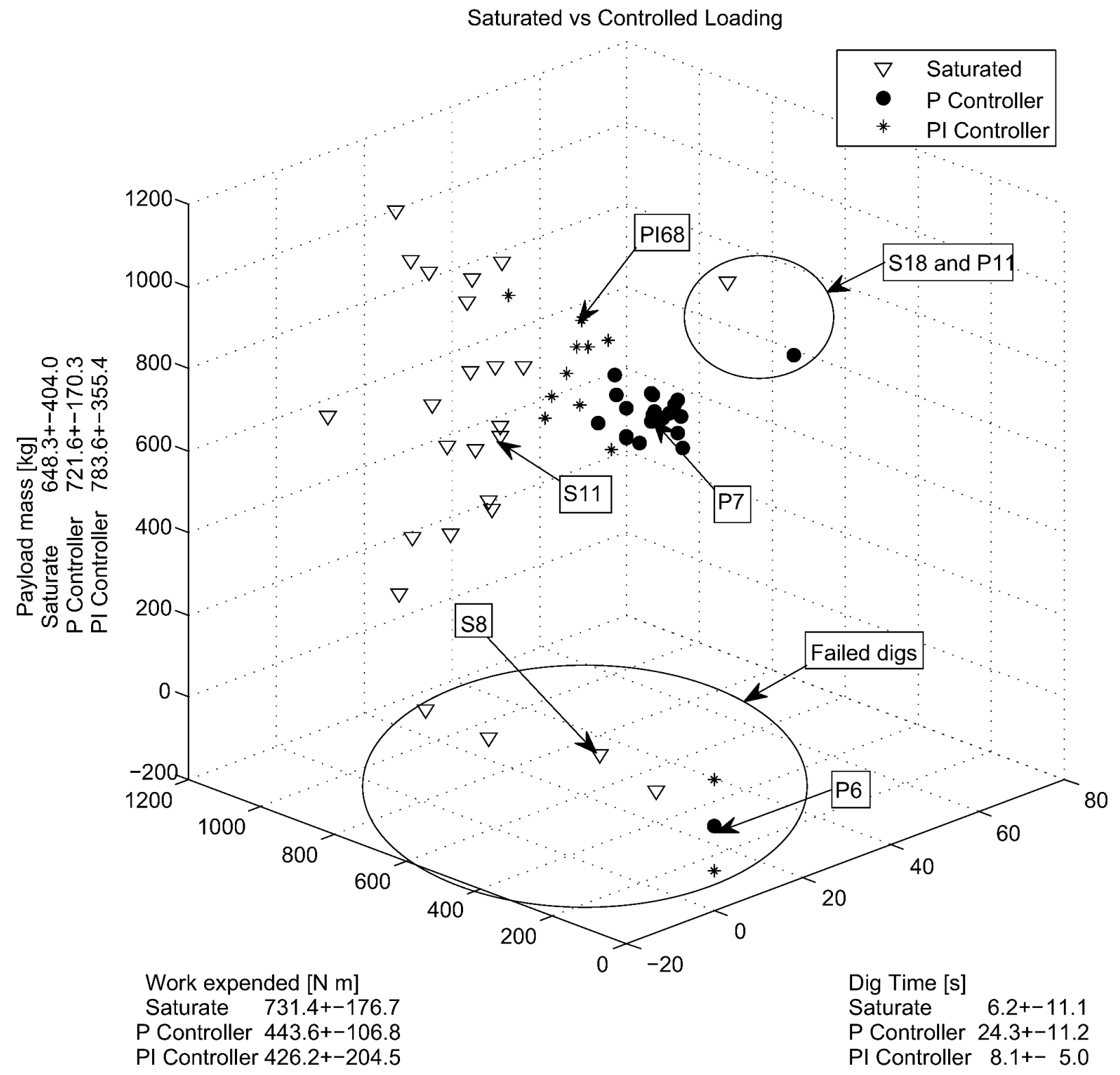

Figure 16: The dig efficiency coordinates for each dig illustrates the relative performance of each controller. The most successful digs are tightly clustered, have low dig time, minimize work expended, and have high mass in the bucket (payload). 
Work and dig time were calculated between entry (after the entry force target is reached), and breakout (when the bucket curls past the breakout target). Work is calculated by using Equation (13), where $W_{d}$ is the work performed by the actuators, $F_{h}$ and $F_{c}$ are the hoist and curl forces in the boom and bucket actuators respectively, and $d_{h}$ and $d_{c}$ are the displacements for each actuator. Let $n$ be the total number of sensor readings and let the subscript $i$ denote the time index associated with each sensor reading. Thus, the total work done $W_{d}$ was estimated by

$$
W_{d}=\frac{1}{2} \sum_{i=1}^{n-1}\left[\left(F_{h, i}+F_{h, i+1}\right) \cdot\left|d_{h, i}-d_{h, i+1}\right|+\left(F_{c, i}+F_{c, i+1}\right) \cdot\left|d_{c, i}-d_{c, i+1}\right|\right] .
$$

Note that this work estimate includes only the work done by the boom and bucket actuators, and not the drivetrain, which thrusts the loader into the pile.

\subsection{R520S Robotic Loading Trials}

A total of $57 \mathrm{dig}$ attempts were made and the dig efficiencies for these digs are shown in Figures 16 and 17. 23 dig attempts where conducted by curling the bucket at a maximum rate after entry (saturated digs), 21 dig attempts where conducted by using the $\mathrm{P}$ admittance controller (Case 1), and 13 dig attempts where conducted by using the PI admittance controller (Case 2). Experienced human operators tend to issue high velocity bucket commands that cause high oscillating forces, which is very similar to the force profiles that result from digging with a saturated controller. Unfortunately, it was not possible to conduct expert manual dig trials because of the modifications made to boom and bucket controls. However, the saturated digs are, in some sense, more interesting because a number of existing patents employ maximum bucket curl in their algorithms.

Five saturated digs, two P digs, and two PI digs failed because entry forces were insufficient for the admittance controllers to take control of the dig. Saturated Dig 18 (S18) and P Dig 11 (P11) took 30 seconds longer than average due to wireless network issues, and low muck pile entry respectively. Dig PI71 achieved a payload of $1018 \mathrm{~kg}$, but took $3 \mathrm{~s}$ longer and required $51 \%$ more work than the average of the PI digs.

\subsubsection{Nominal Digs}

The actuator positions, valve commands, and actuator force plots for the nominal digs (S11, P7, and PI68) are shown in Figure 18. These digs were deemed "nominal" because their dig efficiencies approach the mean dig efficiency for each control case.

In the controlled P dig (Case 1) the admittance controller altered the curl velocity in response to the changing forces resulting in less drastic force changes. The difference between Case 1 and Case 2 is most obvious in these plots since the $\mathrm{P}$ controller (Case 1) only issues curl 


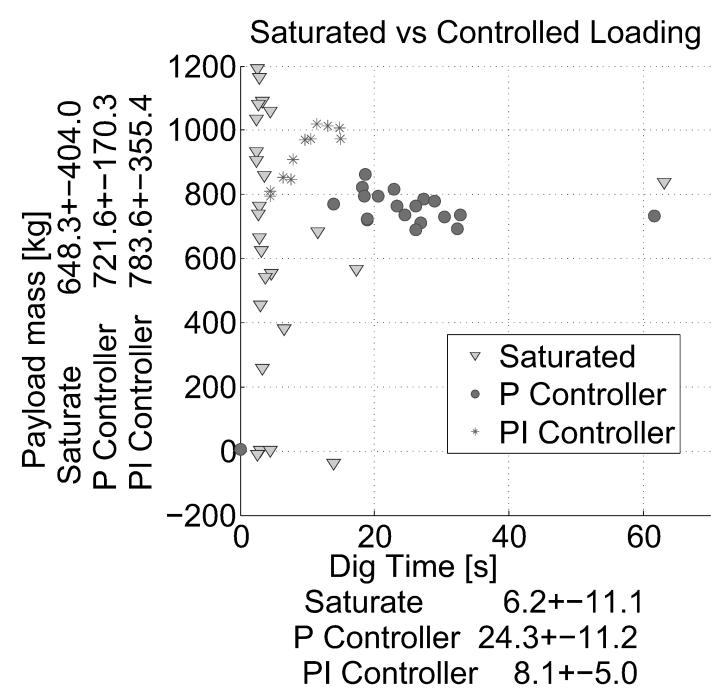

(a) Payload vs. time

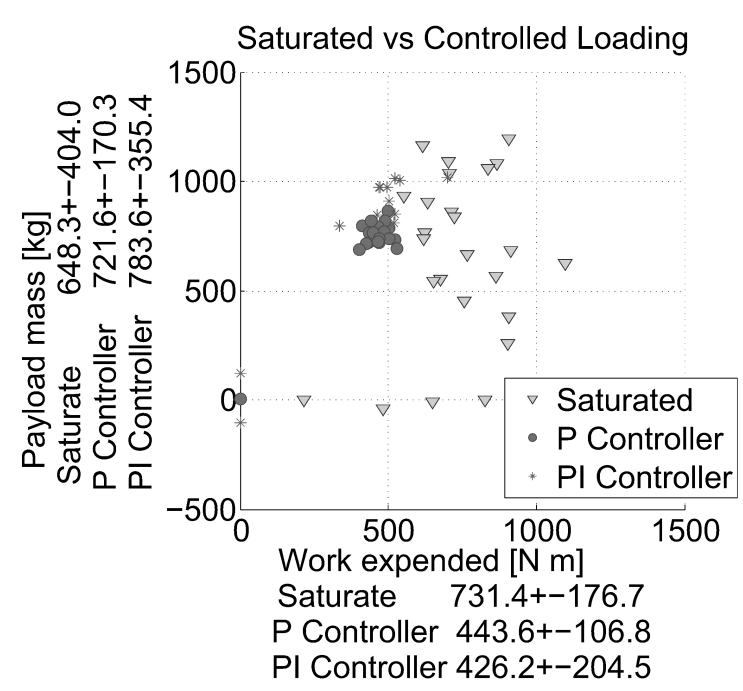

(b) Payload vs. work

Figure 17: Dig efficiency relationships: In (a) most of the saturated digs are clustered around $6 \mathrm{~s}$ while the controlled digs are less clustered and take longer. However, the controlled digs have much less payload variance. In (b) the saturated work readings are much more scattered than the controlled digs, because of uncompensated muck pile reaction forces.

commands when $f_{S}>f_{T}$, and the PI controller (Case 2) only issued upwards curl commands when $f_{S}<f_{T}$. While the PI controller spent more time in the saturated state than the $\mathrm{P}$ controller, this saturation occurred at the beginning and end of each dig when high curl rate is most desirable to prevent stalling in the muck pile. The PI controller exhibited excellent valve response between entry and breakout when the $\mathrm{P}$ controller tended to stagnate. The overall increased velocity of the PI controller resulted in less dig time than the P digs.

\subsubsection{Slow Digs}

The same command and force histories are shown for the slow digs (S18 and P11) in Figures 19(a), and 19(b). The force profile shows the level of force imparted to the pile by the drivetrain when the bucket stopped moving, and the final payload mass indicates that the bucket was filled by the end of the dig. P11 is more interesting because the forces were very close to the $80 \mathrm{kN}$ force target throughout the dig. When the forces rose above the target the admittance controller curled the bucket, which caused the forces to drop and allowed the vehicle to penetrate deeper into the pile. When the loader stalled against the pile the forces rose, and another curl command was sent.

\subsubsection{High Work}

The force histories for an average PI dig (PI68) and the high work dig (PI71) are shown in Figure 20. PI71 takes 3 seconds longer than PI68, which means it has more time steps when calculating work by using Equation (13). The forces in PI71 are also consistently higher 

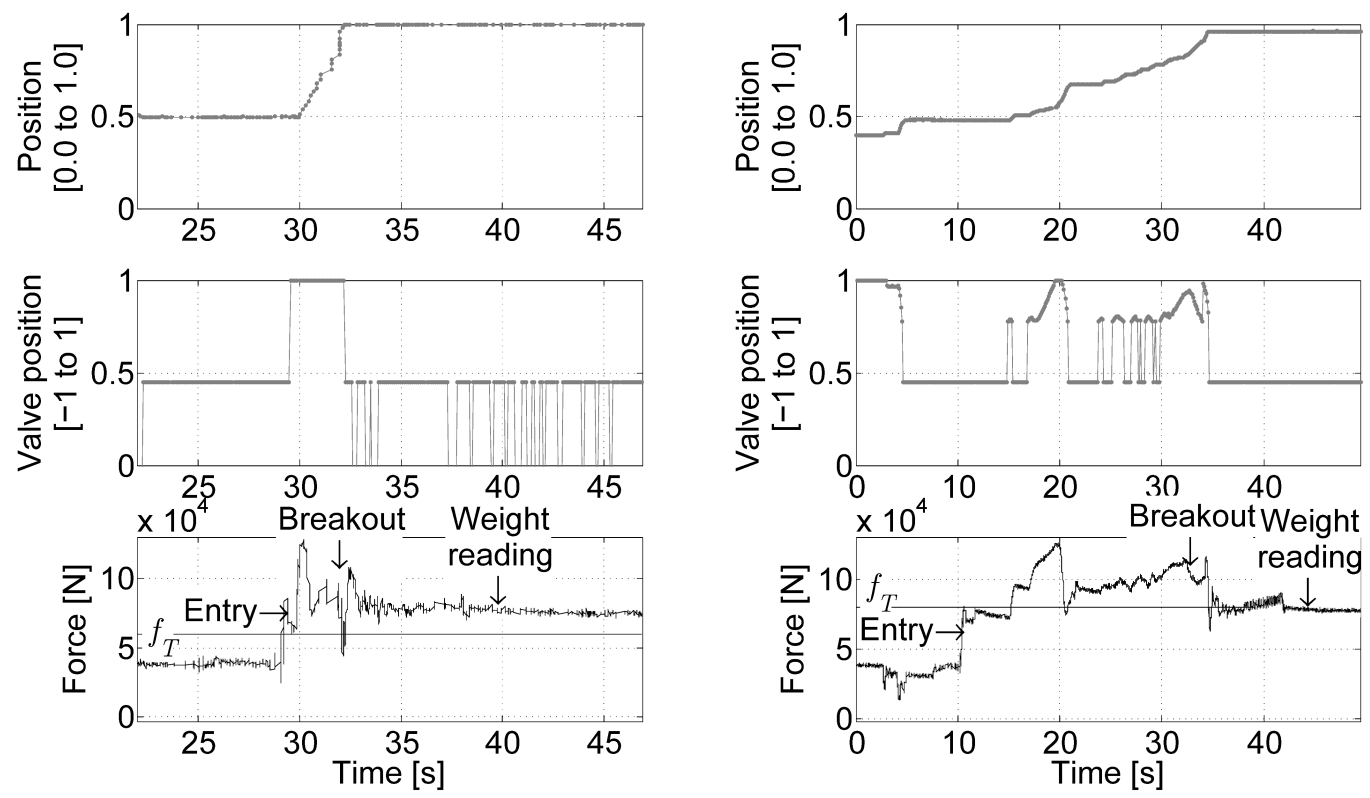

(a) Saturate Dig 11

(b) Controlled P Dig 7
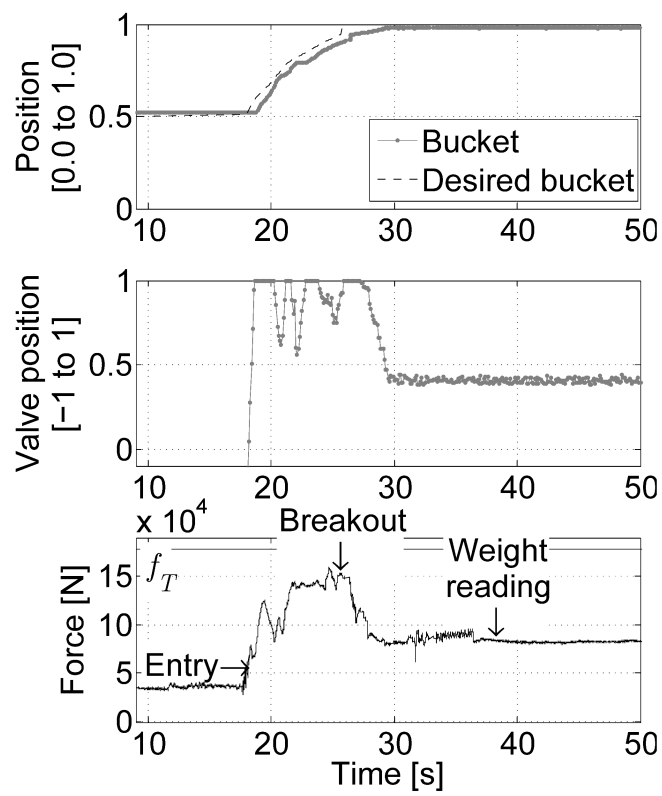

(c) Controlled PI Dig 68

Figure 18: The average dig response for each controller can be used to illustrate how the admittance controller achieves consistent payload despite changing muck pile conditions. (a) The valve commands were full open (saturated) throughout Dig 11, which resulted in the nearly linear response of the bucket actuator. (b) The P controlled dig (Case 1) shows variations in bucket actuator position, and valve commands between 0.5 and 1.0. The bucket also stops moving frequently as the forces approach the $f_{T}$. In (c) the PI controlled dig (Case 2) showed a more fluid valve response to the reaction forces. It was also significantly closer to saturation at entry and breakout without being saturated while digging, which decreased the dig time by $13 \mathrm{~s}$. 

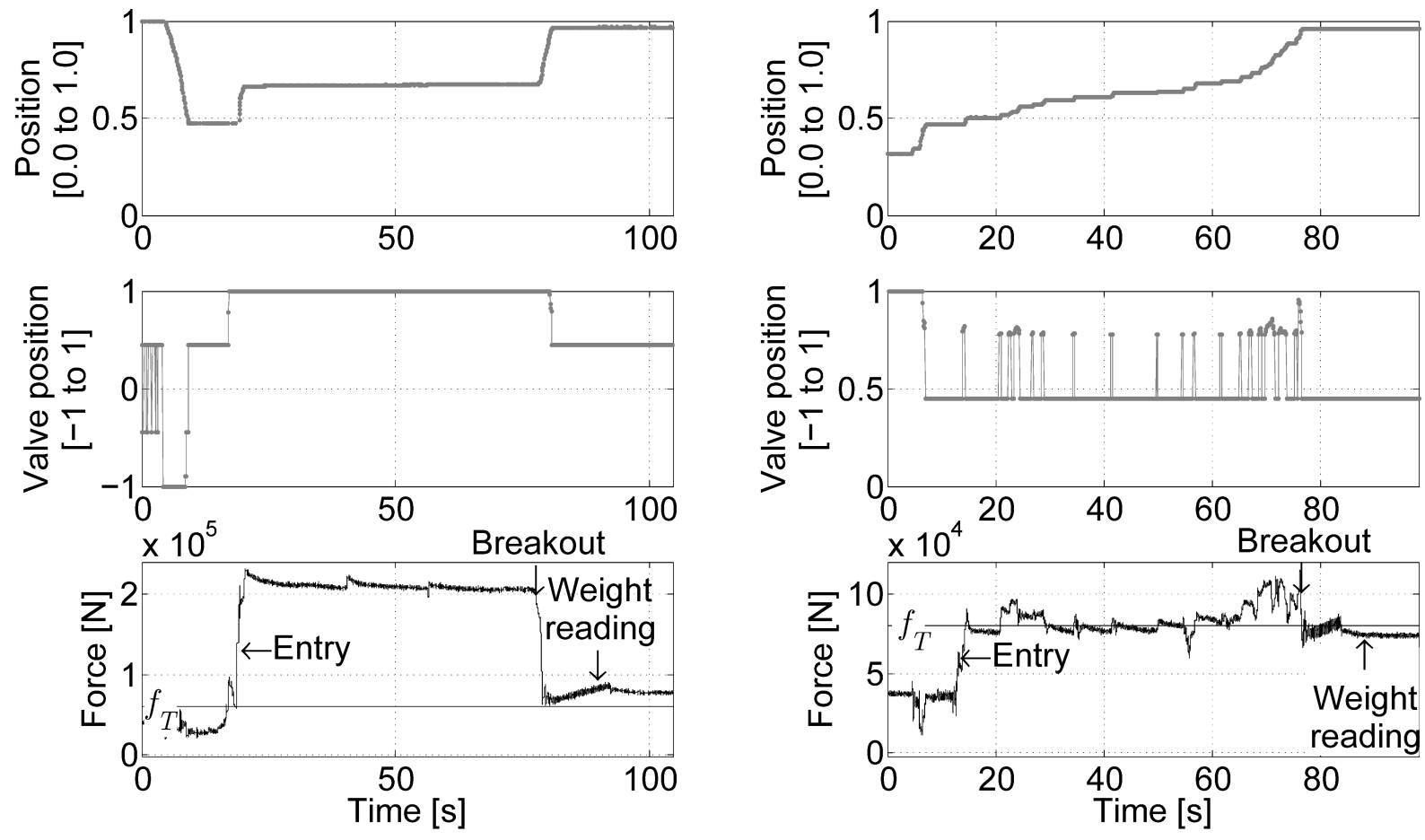

(a) Saturate Dig 18

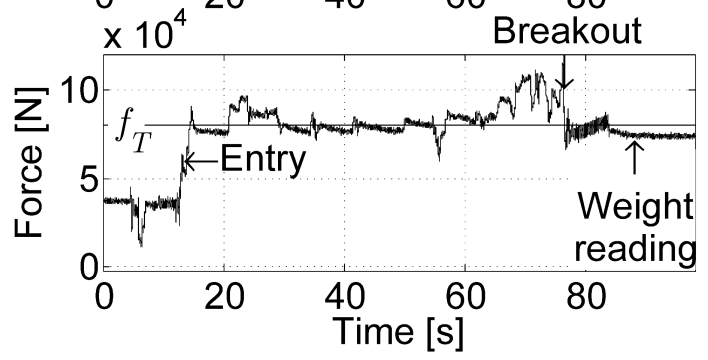

(b) Controlled P Dig 11

Figure 19: The slow digs help illustrate the forward thrust of the loader when the bucket stops curling, and that the admittance controller must be tuned such that the force error never reaches 0.0. (a) The sustained high forces show the level of thrust the drive train can apply to the pile when the bucket is motionless. (b) The reaction forces are close to the target force resulting in brief curl commands when the forces are above the dig target. The resulting curl frees space within the pile for the drive train to briefly advance the loader deeper into the pile. 

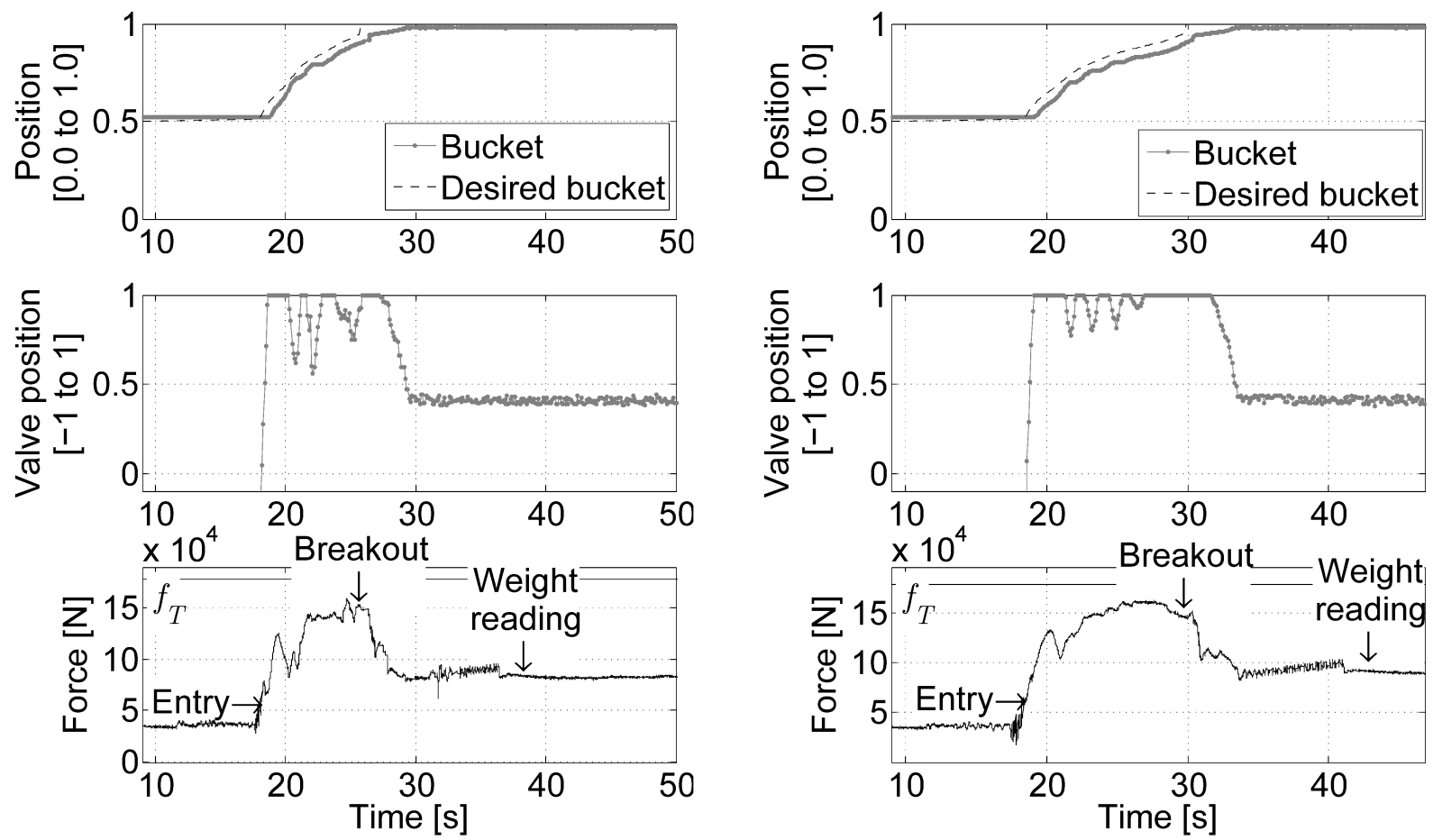

(a) Average work PI Dig 68

(b) High work PI Dig 71

Figure 20: The high work reading can be explained by comparing its dig response to that of an average dig. The average dig (a) takes $3 \mathrm{~s}$ less than the high work dig (b). The high work dig also has higher sustained forces. Both of these factors contribute to the high work dig having double the work of the average dig.

than the forces in PI68. The combination of more time steps and higher sustained forces leads to a $50 \%$ increase in the amount of work required to fill the bucket.

\subsubsection{Failed Digs}

The command and force histories for the failed digs ( $\mathrm{S} 8$ and $\mathrm{P} 6$ ) show that the forces never rose above the entry force, so the bucket controller was never activated, and the digs failed. For brevity, only the force history from P6 is shown in Figure 21. The forces were likely low due to the bucket hitting the ground and unloading the front wheels. The bucket struck the ground due to the poor repeatability of the servos used to control the boom and bucket actuators. Unloading the front wheels decreases the forward thrust and dig reaction forces.

\subsubsection{Dig Efficiency Summary}

The mean \pm one standard deviation dig efficiency values are given in Table 2 for both the complete set of saturated and controlled digs, and for the subset of successful digs. The histograms for all dig efficiency metrics are plotted in Figure 22. The mean dig time rose by $277 \%$ (all digs) and $315 \%$ (successful digs) when the bucket was controlled by the P 


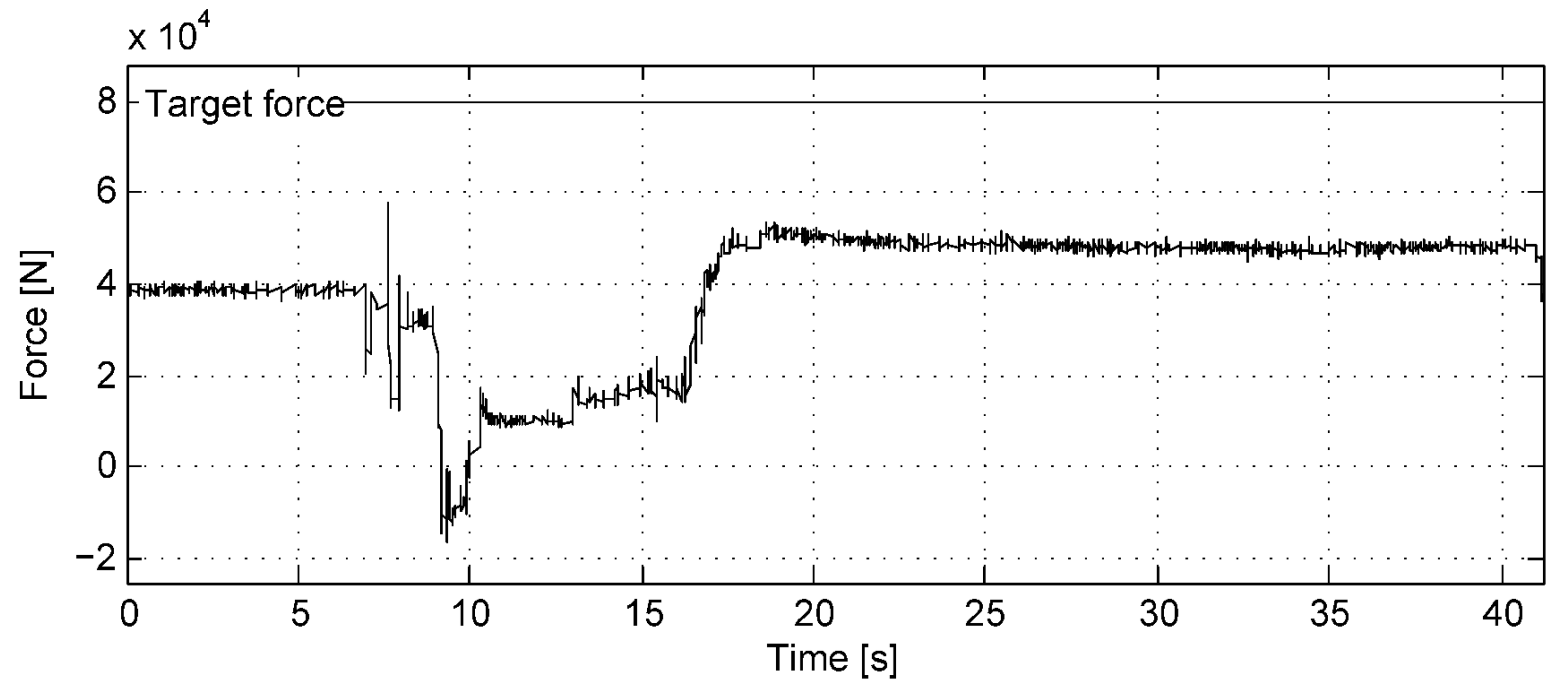

Figure 21: Failed digs occurred when the forces were insufficient to trigger the admittance controller. The forces were likely low because the bucket entered the pile too low, which caused the front axel to lift off the ground (in the video). Low entry unloads the front wheels, which lowers thrust, decreases penetration, and decreases reaction force.

admittance controller compared to when the bucket was moved at its maximum rate. The PI controller reduced dig time over the P controller by $33 \%$ (all digs) and $37 \%$ (successful digs), and had much less variability than either the $\mathrm{P}$ or saturated digs. The $\mathrm{P}$ controlled digs had a $0.3 \%$ increase in payload mass for all digs, but had a $4 \%$ reduction in payload mass when only the successful digs are considered. The PI controller fared better than the saturated and $\mathrm{P}$ controllers with a $10 \%$ (all digs) and $22 \%$ (successful digs) increase in payload mass. The work readings generally increased by $12 \%$ from the saturated to the $\mathrm{P}$ and PI controllers regardless of the set of digs considered. Generally, the successful (all digs that transitioned into the digging phase) controlled digs had much less variability than the saturated digs, and the PI payload was increased by $18 \%$ compared to the saturated digs while dig time only increased by $3 \mathrm{~s}$.

\section{ATLAS COPCO ST14 FIELD EXPERIMENT RESULTS}

For the ST14, ALC tuning tests were used to find final values for the ALC parameters, which were then held constant for all performance tests. These performance tests were conducted to compare the ALC to manual digging. The ALC parameters that were tuned were $f_{T}, k_{A}$, the breakout condition, as well as the entry and weighing poses. Additionally, field tuning revealed key information about controller saturation, and ground detection. 

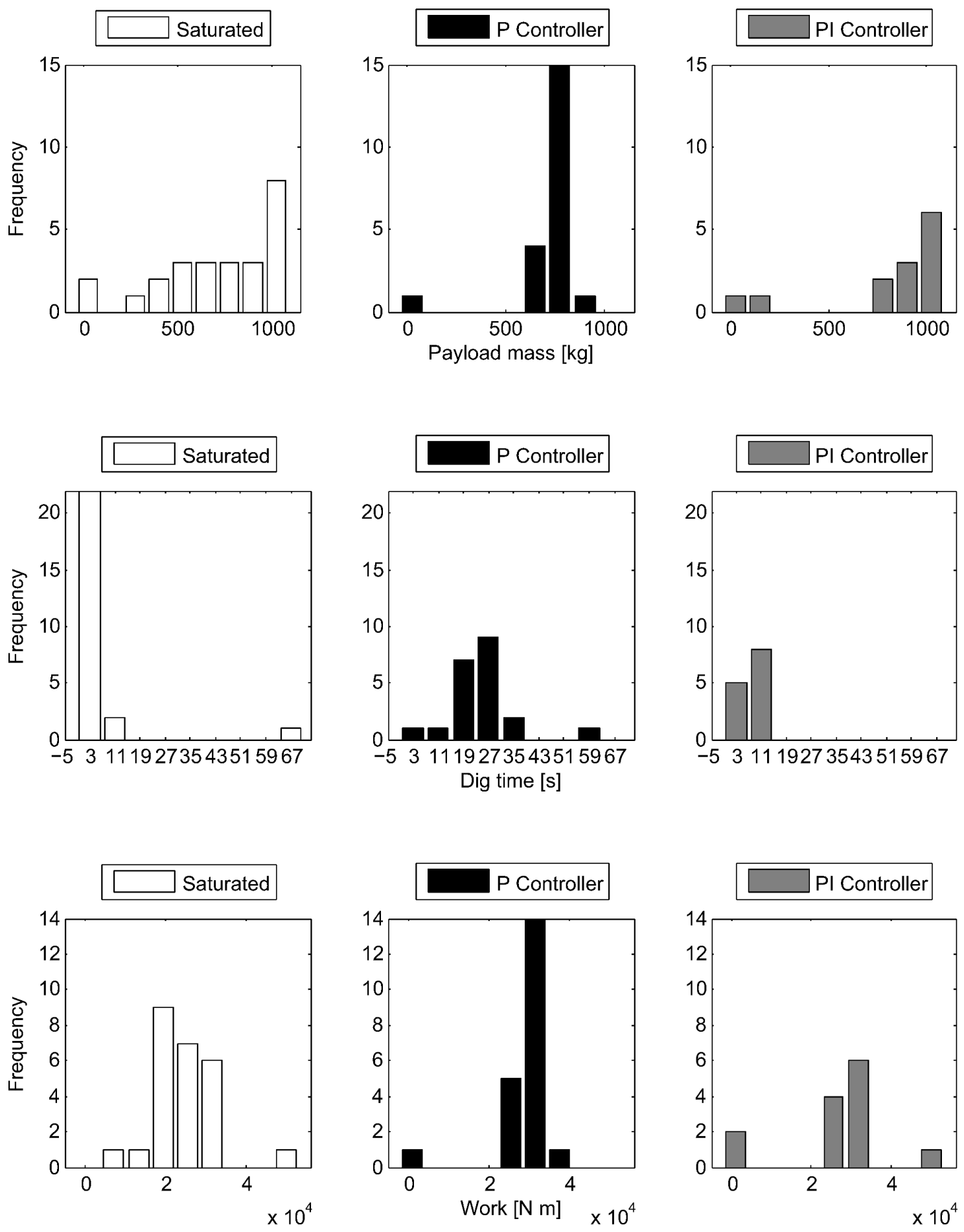

Figure 22: The histograms for all R520S digs using each controller help illustrate the superior consistency of the admittance controllers over the fully saturated controller. The variance of both the payload and work readings are much smaller for the controlled digs than the saturated digs especially when the failed digs are removed. 
Table 2: Saturated and controlled dig efficiency averages.

\begin{tabular}{lcrcrc}
\hline All digs & \multicolumn{5}{c}{} \\
\hline$\epsilon_{d}$ & Saturated & P vs. Sat. & P Controller & PI vs. Sat. & PI Controller \\
\hline$t_{d}[\mathrm{~s}]$ & $6.5 \pm 12.1$ & $+276.9 \%$ & $24.5 \pm 11.3$ & $+6.5 \%$ & $8.1 \pm 5.0$ \\
$W_{d}[\mathrm{~N} \mathrm{~m}]$ & $24458 \pm 8495$ & $+15.1 \%$ & $28145 \pm 6711$ & $+10.1 \%$ & $27287 \pm 14511$ \\
$M_{d}[\mathrm{~kg}]$ & $721 \pm 326$ & $-0.3 \%$ & $719 \pm 170$ & $+9.9 \%$ & $792 \pm 335$ \\
\hline Without failed digs & \multicolumn{5}{c}{} \\
\hline$t_{d}[\mathrm{~s}]$ & $6.2 \pm 12.6$ & $+314.5 \%$ & $25.7 \pm 10.1$ & $+13.2 \%$ & $9.6 \pm 3.8$ \\
$W_{d}[\mathrm{~N} \mathrm{~m}]$ & $25658 \pm 8697$ & $+15.2 \%$ & $29551 \pm 1929$ & $+22.3 \%$ & $32.46 \pm 8766$ \\
$M_{d}[\mathrm{~kg}]$ & $784 \pm 254$ & $-3.7 \%$ & $755 \pm 40$ & $+18.5 \%$ & $924 \pm 85$ \\
\hline
\end{tabular}

\subsection{Force Target $f_{T}$}

Figure 23 shows the ALC digging response as $f_{T}$ was reduced from $11 \mathrm{MN}$ to $9 \mathrm{MN}$. An initial guess for $k_{A}$ was 0.001 , which was selected by using

$$
k_{A} \approx r \cdot \frac{v_{A_{\max }}}{f_{S_{\max }}}
$$

where $r=\frac{1}{8}, v_{A \max }$ is the maximum bucket actuator velocity $(0.08 \mathrm{~m} / \mathrm{s})$, and $f_{S_{\max }}$ is the maximum force sensed in the boom actuator $(10 \mathrm{MN}) . r$ is an arbitrary scalar that sets the minimum increment between no gain and a gain that results in complete actuator saturation. Initial tuning results (in Section 6.2) indicated that the controller was unacceptably saturated when $k_{A}$ was increased to 0.002 . Saturation should be avoided because it means the admittance controller is no longer maintaining the desired admittance dictated by Equation (7). The manual results (in Section 6.4, Figure 26) show that digging without compensating for the digging forces tends to result in less overall payload and more payload variability.

Decreasing the dig target increased dig time, decreased bucket velocity, and decreased bucket actuator control valve saturation. When $f_{T}$ dropped to $9.5 \mathrm{MN}$, the dig time increased from 8 to $30 \mathrm{~s}$, the bucket velocity was much slower, and the sensed forces were barely high enough to bias the admittance controller toward the breakout condition. At $f_{T}=9.0 \mathrm{MN}$ these effects became so severe that the dig failed because the ALC could then reduce the force error $e_{f}$ close to zero. Figures 23(a) and 23(b) also illustrate that more controller saturation leads to higher, more irregular forces. The 11-MN and 10-MN test results indicate that these irregular forces generated higher payloads, but also more payload variability. It should also be noted that for the 11-MN and 10-MN tests the bucket curls down (see between 10 and $15 \mathrm{~s}$ ) when the boom forces exceed their respective $f_{T}$ values. While curling down may seem counter productive, it allows the bucket to circumvent force concentrations and dig deeper into the pile. We believe that this results in increased payload and less payload variability because the admittance between the bucket and muck pile is maintained, and hence each dig trajectory is tailored to the unique force environment encountered within the pile. 

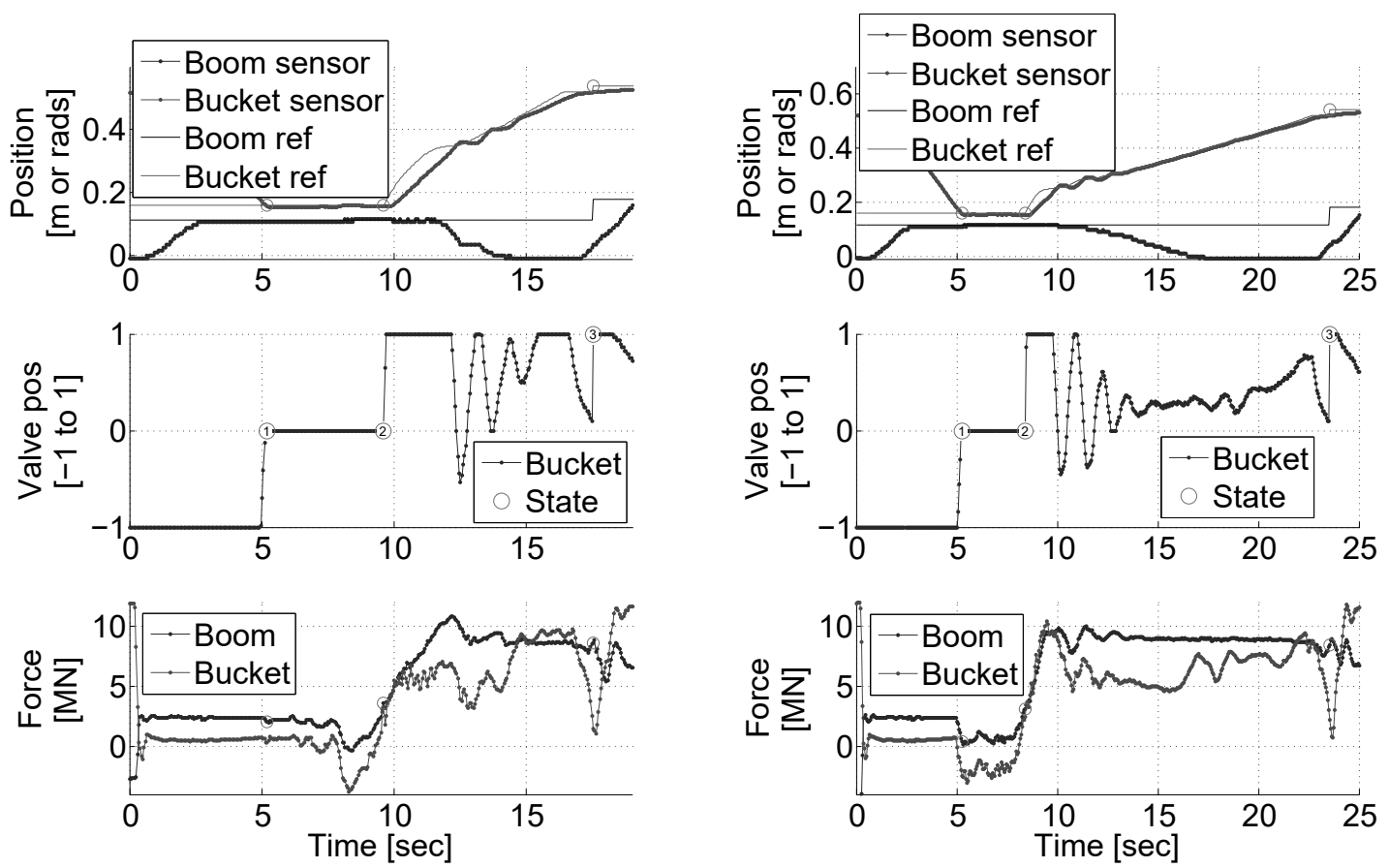

(a) $11.0 \mathrm{MN}$ dig target

(b) $10.0 \mathrm{MN}$ dig target
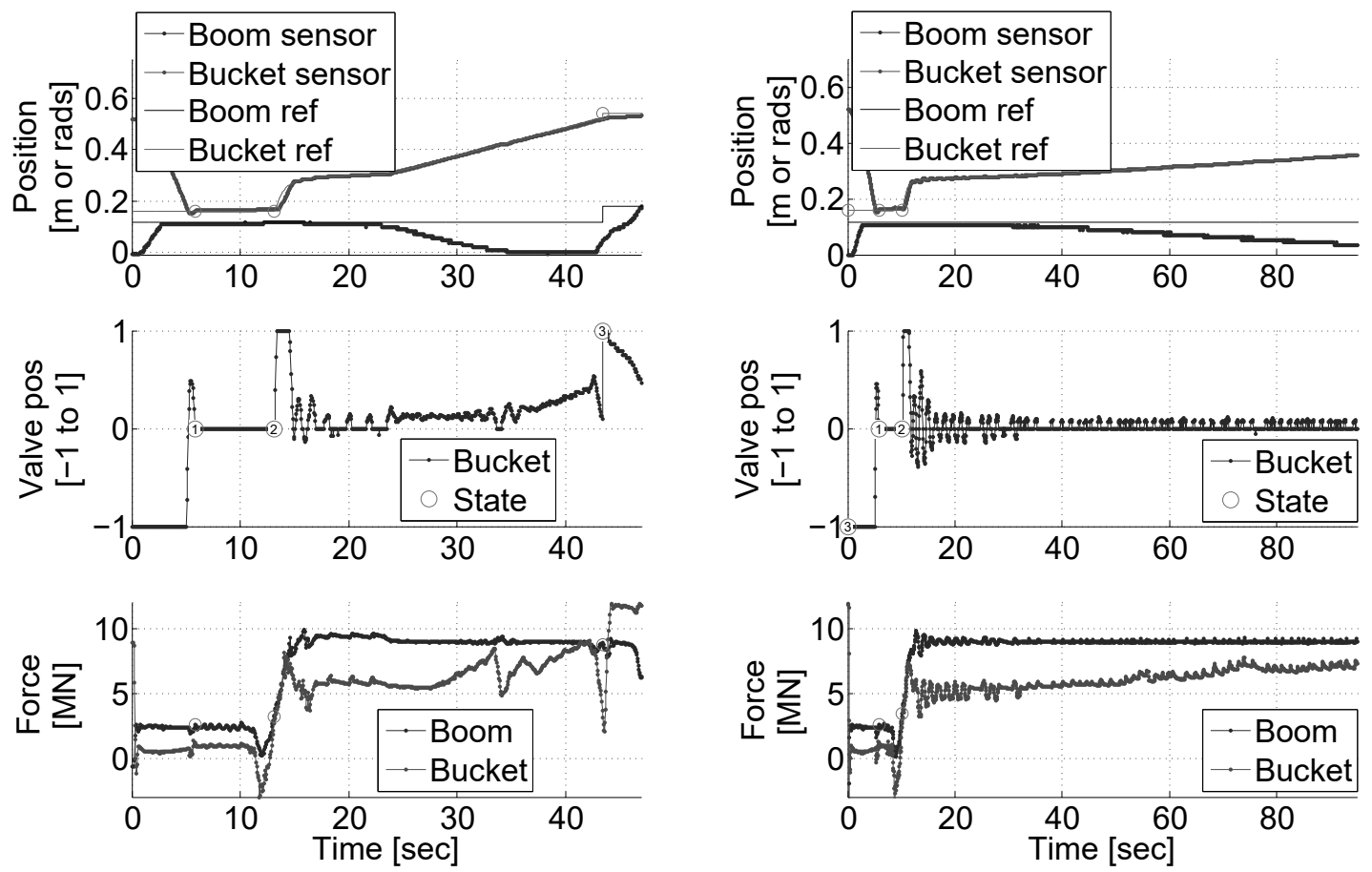

(c) $9.5 \mathrm{MN}$

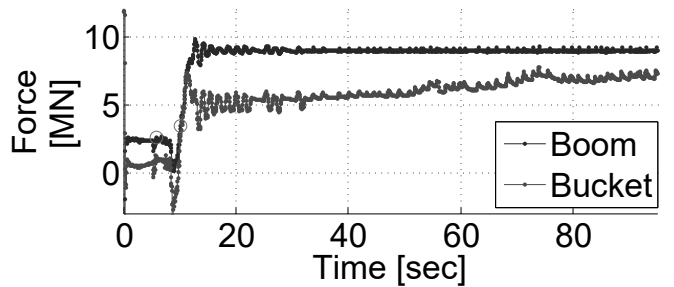

(d) $9.0 \mathrm{MN}$ dig target

Figure 23: At $11 \mathrm{MN}$ (a) the ALC was more saturated than in the $10 \mathrm{MN}$ digs (b), but both completed successfully. The $10 \mathrm{MN}$ digs took twice as long as the $11 \mathrm{MN}$ digs, and the 9.5 MN digs (c) took three times longer than the $11 \mathrm{MN}$ digs. At $9 \mathrm{MN}$ (d), the dig failed because the ALC was able to reduce the error to 0.0 and the curl rate dropped too low for the ALC to finish in a reasonable time. 

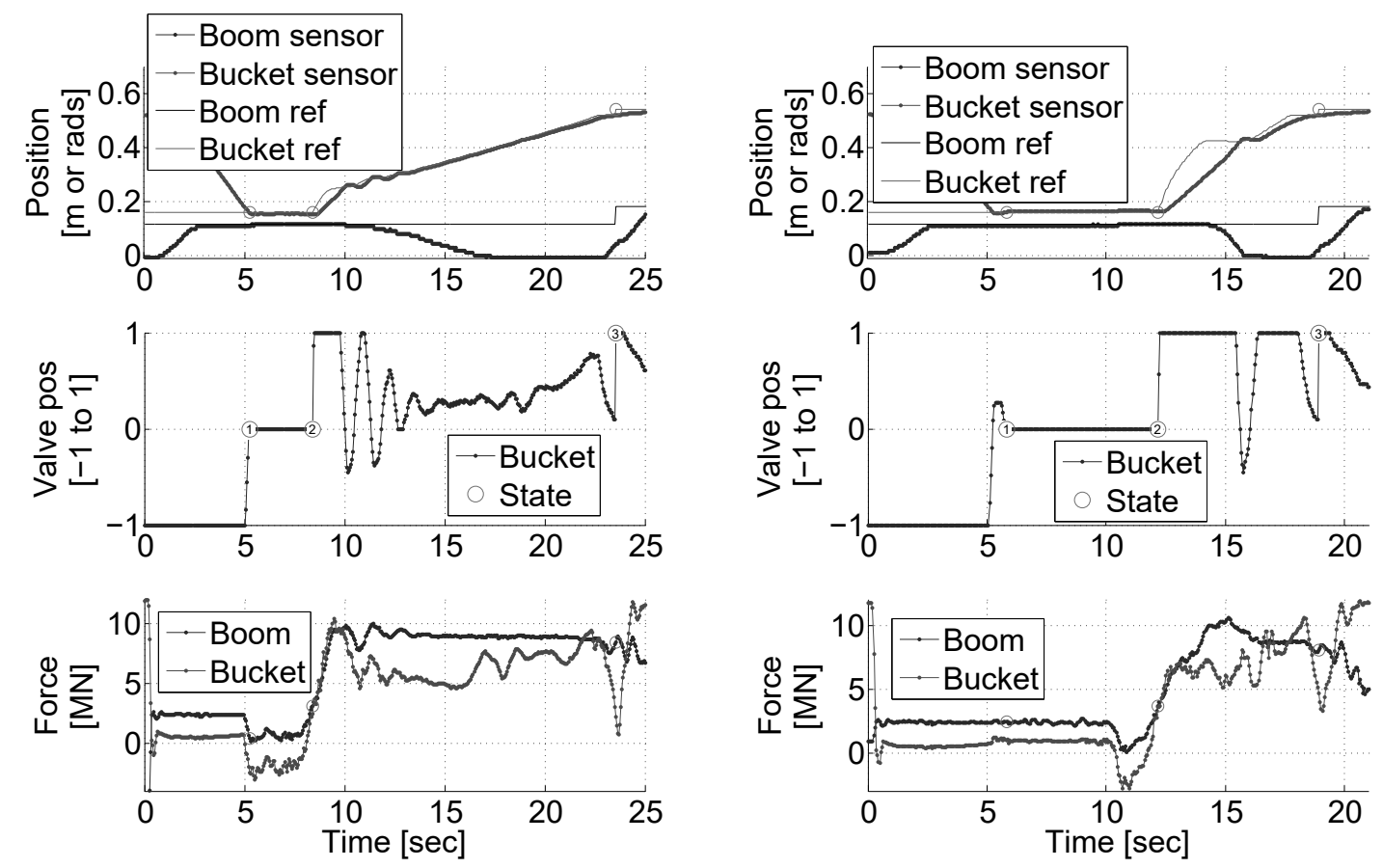

(a) ALC gain at 0.001 , Force target $10 \mathrm{MN}$

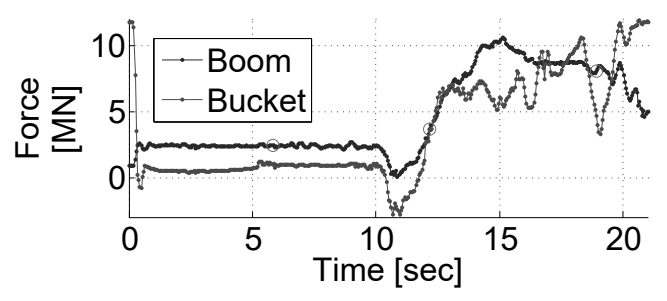

(b) ALC gain at 0.002 , Force target $10 \mathrm{MN}$

Figure 24: ALC gain selection - The ALC gain at 0.001 (a) issues excellent valve commands with little saturation compared to the 0.002 gain (b), which was almost always saturated.

\subsection{Admittance Gain $k_{A}$}

Figure 24 shows the ALC responses when the admittance gain $k_{A}$ was raised from 0.001 to 0.002 while $f_{T}$ was maintained at $10 \mathrm{MN} . k_{A}=0.002$ was too high since the ALC valve commands were almost always saturated. $k_{A}=0.001$ was used for both the $10-\mathrm{MN}$ and 11-MN performance tests, and was high enough to cover both positive and negative valve command ranges without saturation.

\subsection{Weighing Pose Entry Pose and Breakout Condition}

The weighing pose was set such that the bucket was in free space above the pile was tilted downwards at approximately $15^{\circ}$, and scraping the floor. The breakout condition was set to $0.500 \mathrm{~m}$ of bucket actuator extension because the bucket is prevented from curling further by stops on the boom arms. However, as the boom rises these stops move further back. Midway through the tuning process, the bucket breakout extension was increased from 0.500 to $0.520 \mathrm{~m}$, which increased payload to $12.50 \mathrm{t}$ from $10.13 \mathrm{t}$. This increase occurred because the bucket curled back farther as soon as the boom started to lift, which kicked more material into the bucket. 16 runs were made at the $10-\mathrm{MN}$ dig target force and 10 runs were made at the 11-MN dig target force while all other parameters were kept constant so that the ALC could be compared to a manual operator. 


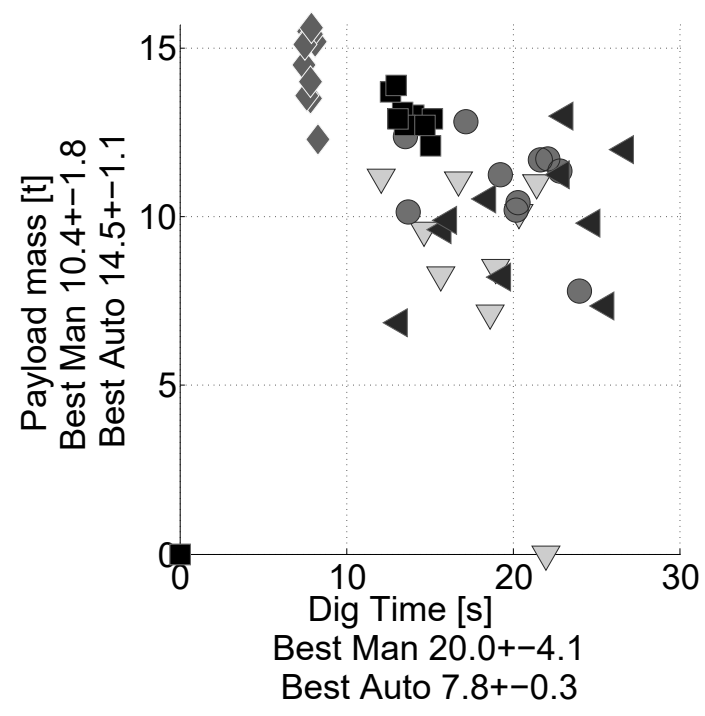

(a) Payload vs. Time

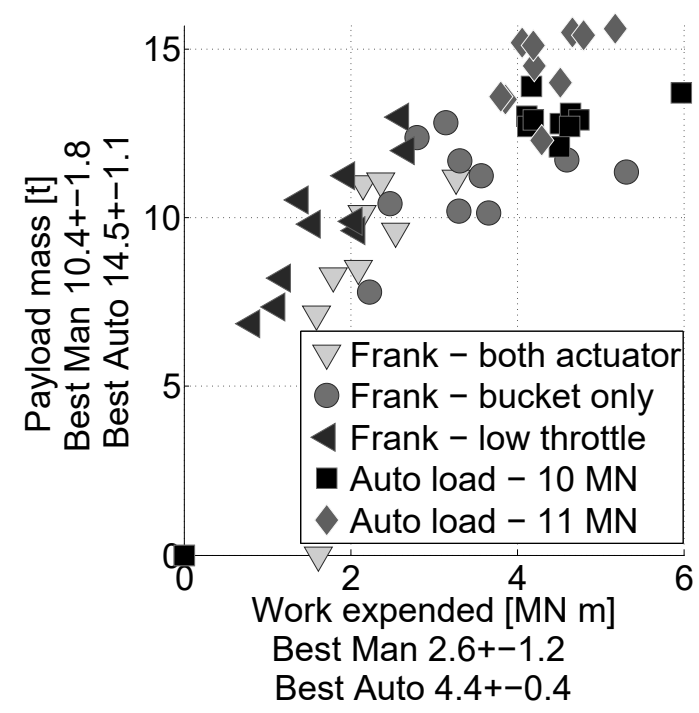

(b) Payload vs. Work

Figure 25: The payload vs dig time (a) and the payload vs work (b) dig efficiency plots show that the only autonomous dig attempts that were less than $12 \mathrm{t}$ were the six $10 \mathrm{MN}$ dig target digs that failed due to insufficient entry force to trigger the admittance controller. The manual dig attempts had much greater variability in payload mass, dig time, and actuator work than the tightly clustered autonomous dig attempts.

\subsection{ALC Performance}

The dig efficiency results from the 26 autonomous and 28 manual digs are shown in Figure 25. The number of tests was dictated by the availability of the apparatus, operator, and test site. The manual digs with the highest dig efficiencies were Frank's bucket-only, and low-throttle digs. The autonomous digs with the highest efficiencies were the 11-MN digs. While the 10-MN autonomous digs were also excellent, six of these digs failed. The likely cause of these failures was low entry force due to striking the ground or spillage before entry.

Figure 25(a) shows the payload and dig times for the 54 dig attempts. While the autonomous dig attempts were tightly clustered, there was much more variability in the manual dig times and payloads. Figure 25(b) shows the payload and work expended for the same 54 dig attempts. The autonomous dig attempts were again tightly clustered, while there was much more variability in the manual digs. Work also increased as payload increased. All dig efficiency results are summarized in Table 3.

Figure 26 shows the results for an excellent manual, and typical autonomous dig attempt. In both digs only the bucket was actuated either by Frank or by the ALC. Frank oscillated the bucket rhythmically while the ALC only oscillated when the forces were below the $11 \mathrm{MN}$ target force. This reduced oscillation resulted in smoother force and valve command profiles, and ultimately greater bucket velocity control, and more payload in less time.

Tests were conducted in both a settled and an unsettled muck pile, as well as the two muck 
Table 3: The ALC loaded $39 \%$ more payload in $61 \%$ less time, but required $68 \%$ more work than the best expert operator digs. The ALC was also much more consistent.

\begin{tabular}{lccc}
\hline$\epsilon_{d}$ & Manual & Autonomous & Difference \\
\hline$t_{d}[\mathrm{~s}]$ & $20.03 \pm 4.10$ & $7.82 \pm 0.26$ & $-61 \%$ \\
$W_{d}[\mathrm{MN} \mathrm{m}]$ & $2.59 \pm 1.17$ & $4.36 \pm 0.43$ & $+68 \%$ \\
$M_{d}[\mathrm{t}]$ & $10.41 \pm 1.77$ & $14.47 \pm 1.09$ & $+39 \%$ \\
\hline
\end{tabular}
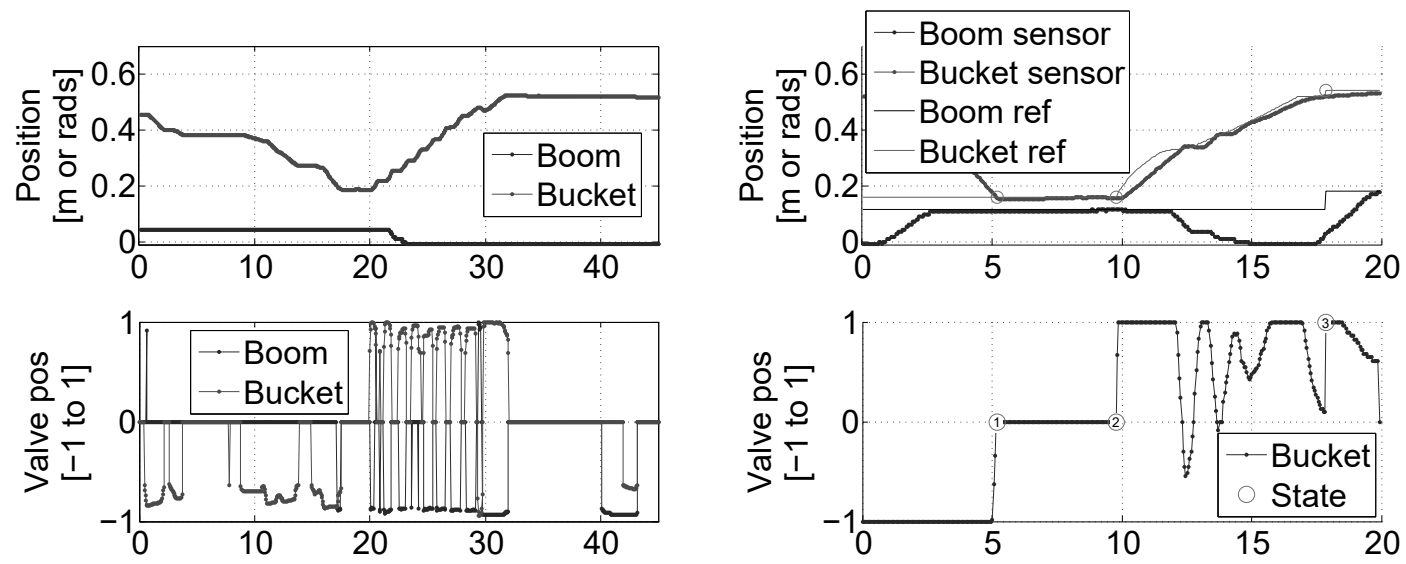

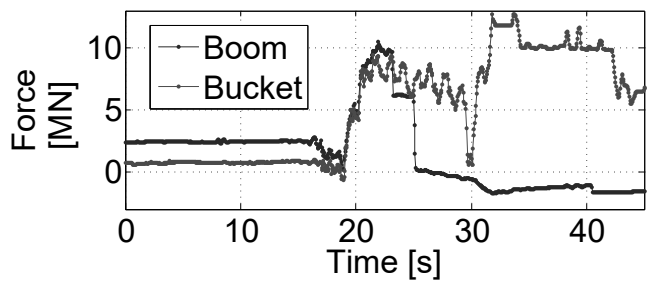

(a) Manual (Frank)

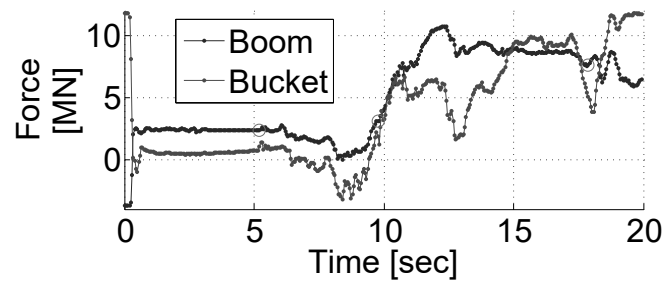

(b) Autonomous (11 MN)

Figure 26: In (a) Frank gave the bucket regular oscillating command signals that resulted in a jagged force profile, and severe valve position oscillations between 1 and 0 . The ALC in (b) sent smoother commands that used partial valve positions to regulate the speed of the bucket. As a result, the forces were smoother than the manual dig attempts. Additionally the bucket curled down at $12 \mathrm{~s}$ to reduce the forces below the dig target. This behaviour caused the bucket to dig deeper into the pile, and ultimately increased the final payload. 
piles with different rock types and size distributions. The average payload in the unsettled pile was $14.47 \pm 1.09 \mathrm{t}$ (11-MN autonomous tests). Only one test was conducted in the settled pile (due to the time it takes for settling), which yielded $12.50 \mathrm{t}$. The rock type and size distribution had little effect on the ALC because the force profiles resulting from digging in the two piles were nearly identical. The payload change was slight, going from $11.40 \mathrm{t}$ in the lower density $0.20 \pm 0.09 \mathrm{~m}$ limestone rock in Drift 159, to an average of $12.93 \pm 0.55 \mathrm{t}$ in the higher density $0.48 \pm 0.19 \mathrm{~m}$ granite rock in Drift 165 .

\section{CONCLUSION}

Modern LHDs used in many mining operations can now haul and dump autonomously, but still require an operator to load the bucket with fragmented rock from muck piles. Despite a long history of research in the field of robotic excavation, a commercial viable system for autonomously loading fragmented rock has yet to materialize. In this paper, we describe the design, implementation and testing of a a new admittance-based approach to autonomous loading. Three admittance-based controllers were incorporated into an autonomous loading algorithm and field tested by using two representative vehicles, three muck piles, and two different testing environments. The admittance-based controllers performed well in a variety of muck pile conditions and were able to circumvent force concentrations indicative of subsurface obstacles. The tuning process was similar for both vehicles despite their dissimilar size, power, environments, and muck piles. This result is very encouraging given the involved tuning and development process of other autonomous digging methods. It is worth noting however that both platforms had similar hoist and curl mechanisms.

Successful digs were also more consistent and more productive compared with simply allowing the bucket to curl at its maximum rate, or having a human operator perform the loading operation. For instance the PI version of the admittance controller deployed on a surface loader (Case 2) was able to load $18 \%$ more material than curling the bucket at its maximum rate, and the $\mathrm{P}$ version of the admittance controller deployed at the Kvarntorp Mine on an Atlas Copco ST14 LHD (Case 3) was able to load $39 \%$ more payload than a skilled human operator. While most dig attempts took less than $35 \mathrm{~s}$, the $\mathrm{P}$ version (Case 1) of the admittance controller took 18 s longer than the fully saturated controller. This extra dig time was mitigated at the cost of increased variability by using the PI version (Case 2) of the admittance controller. By contrast, the Case $3 \mathrm{P}$ version of the admittance controller reduced dig time in the more challenging underground mine environment by $12.2 \mathrm{~s}$ compared to the human operator. The work expended while using the admittance controller generally increased due to loading more material than when the saturated controller or the human operator were loading. It should also be noted that the $610 \mathrm{MN}$ digs that failed could have been avoided by using a more robust means for detecting muck pile entry. One such method could be by using a ranging sensor to detect the relative location of the bucket and the toe of the muck pile. 
One might also build on the proposed robotic loading algorithm to see whether it can be improved by implementing an adaptive admittance controller - e.g., similar to the method of Seraji (1994) — or to improve tuning by developing an iterative learning controller, as described for other applications in Bristow, Tharayil, and Alleyne (2006) and Ahn, Moore, and Chen (2007). However, it should be noted that 800 to $900 \mathrm{~kg}$, and 13.4 to $15.6 \mathrm{t}$ are approaching the payload limits for both the R520S surface loader, and the ST14. Other future work could include implementing the ALC in a more encompassing digging algorithm that tackles the entry point selection, and overall muck pile removal problems.

\section{Acknowledgments}

The authors would like to thank Brian Lynch, Chris McKinnon, Michal Pasternak, Jordan Mitchell, and Oscar Rielo at Queen's University, as well as Curtis Watson and the engineering team that built our much more practical R520S bucket. Thanks also to Mike Purvis, Ryan Gariepy, and the Clearpath Robotics team for support with some of the hardware and software used in the setup and execution of the Kubota-based experiments described in this paper. Special thanks to Ola Pettersson, Morgan Rody, and Jörgen Appelgren at Atlas Copco Rock Drills AB for facilitating access to the Kvarntorp underground testing facility, an ST14 LHD machine, and for travel and living support during field work in Sweden.

This research was financially supported in part by the Natural Sciences and Engineering Research Council of Canada (NSERC) under Discovery Grant number 371452-2009 and by Atlas Copco Rock Drills AB, Örebro, Sweden.

\section{References}

Ahn, H.-S., Moore, K. L., \& Chen, Y. (2007). Iterative learning control: robustness and monotonic convergence for interval systems. Springer.

Almqvist, H. (2009). Automatic bucket fill (MSc.). University in Linköping: Tekniska Högskola, Linköping, Sweden.

Alshaer, B. J., Ingram, R. G., Krone, J. J., Berry, J. K., \& Harris, J. J. (2009, July 7). U.S. Patent No. 7555855, Automatic digging and loading system for work machine. Peoria, Ill: U.S. Patent and Trademark Office.

Atlas Copco. (2012, July). Atlas copco underground loaders: Scooptram st14 technical specification. Atlas Copco. ([Online]. Available: http://www.atlascopco.ca/images/ technical_specification_scooptram_st14_9851_2350_01_tcm836-1532858.pdf)

Bernold, L. (1993, January). Motion and path control for robotic excavation. Journal of Aerospace Engineering, 6(1), 1-18.

Blackwell, G. (Ed.). (2013). Remote and semi-automated operation of an electric cable shovel. Montréal, Canada: Proceedings of the 30th ISARC.

Bradley, D. A., \& Seward, D. W. (1998, March). The development, control and operation of an autonomous robotic excavator. Journal of Intelligent and Robotic Systems, 21(1), 
73-97.

Bristow, D., Tharayil, M., \& Alleyne, A. (2006). A survey of iterative learning control. Control Systems, IEEE, $26(3), 96-114$.

Dasys, A., Geoffroy, L., \& Drouin, A. (1999, August 24). U.S. Patent No. 5941921, Sensor feedback control for automated bucket loading. Canada: U.S. Patent and Trademark Office.

Dobson, A., Marshall, J., \& Larsson, J. (2015, June). Admittance control for robotic loading: Underground field trials with an lhd. In Proceedings of the 10th conference on field and service robotics (Vol. 10, p. 14). Toronto, Canada.

$\mathrm{Fu}$, T. (2011). Multirobot lunar excavation using an artificial-neural-tissue controller (MASc). University of Toronto Institute for Aerospace Studies, 4925 Dufferin Street, Toronto, Ontario, Canada M3H 5T6.

Ha, Q., Santos, M., Nguyen, Q., Rye, D., \& Durrant-Whyte, H. (2002). Robotic excavation in construction automation. IEEE: Robotics and Automation Magazine, 9(1), 20-28.

Hemami, A., \& Daneshmend, L. (1992, May). Force analysis for automation of the loading operation in an lhd-loader. In Robotics and automation, 1992. proceedings., 1992 ieee international conference on (p. 645-650 vol.1). doi: 10.1109/ROBOT.1992.220219

Hemami, A., \& Hassani, F. (2009). An overview of autonomous loading of bulk material. In Proceedings of the 26th international symposium on automation and robotics in construction (pp. 405-411).

Johnson, D. A., Sahlin, M. P., Anderson, E. R., \& Cherney, M. J. (2010, December 14). U.S. Patent No. 7853384, Method and system for controlling a vehicle for loading or digging material. Moline, Ill: U.S. Patent and Trademark Office.

Lever, P. (2001, December). An automated digging control for a wheel loader. Robotica, $19(5), 497-511$.

Maeda, G. J., Rye, D. C., \& Singh, S. P. N. (2013). Iterative autonomous excavation. In K. Yoshida \& S. Tadokoro (Eds.), Field and service robotics (Vol. 92, p. 369-382). Berlin Heidelberg Germany: Springer Berlin Heidelberg.

Marshall, J. A. (2001). Towards autonomous excavation of fragmented rock: Experiments, modelling, identification and control (Master of Science: Engineering). Queen's University, Kingston, On. Canada.

Marshall, J. A., Barfoot, T. D., \& Larsson, J. (2008, June). Autonomous underground tramming for center-articulated vehicles. Journal of Field Robotics, 25 (6-7), 400-421.

Marshall, J. A., Murphy, P. F., \& Daneshmend, L. K. (2008, July). Toward autonomous excavation of fragmented rock: full-scale experiments. IEEE Transactions on Automation Science and Engineering, 5(3), 562-566.

McKinnon, C., \& Marshall, J. A. (2014, July). Automatic identification of large fragments in a pile of broken rock using a time-of-flight camera. IEEE Transactions on Automation Science and Engineering, 11(3), 935-942.

Petty, M., Billingsley, J., \& Tran-Cong, T. (1997). Autonomous lhd loading.

Richardson-Little, W., \& Damaren, C. (2008, January). Position accommodation and compliance control for robotic excavation. Journal of Aerospace Engineering, 21 (1), 27-34.

Rocke, D. J. (1996, June 25). U.S. Patent No. 5528843, Control system for automatically 
controlling a work implement of an earthworking machine to capture material. Peoria, Ill: U.S. Patent and Trademark Office.

Rocke, D. J. (1997, October 28). U.S. Patent No. 5682312, Self-adapting excavation control system and method. Peoria, Ill: U.S. Patent and Trademark Office.

Salcudean, S., Tafazoli, S., Hashtrudi-Zaad, K., \& Lawrence, P. (1998). Evaluation of impedance and teleoperation control of a hydraulic mini-excavator. Lecture Notes in Control and Information Sciences, 232, 229-240.

Sarata, S., Koyachi, N., \& Sugawara, K. (2008, September). Field test of autonomous loading operation by wheel loader. Acropolis convention center, Nice, France: Proceedings of the IEEE/RSJ International Conference on Intelligent Robots and Systems.

Schmidt, D., Proetzsch, M., \& Berns, K. (2010, May). Simulation and control of an autonomous bucket excavator for landscaping tasks. Proceedings of the IEEE International Conference on Robotics and Automation.

Seraji, H. (1994). Adaptive admittance control: an approach to explicit force control in compliant motion (Vol. 4). Proceedings of the IEEE International Conference on Robotics and Automation.

Shi, X., Lever, P., \& Wang, F. (1998). Autonomous rock excavation: Intelligent control techniques and experimentation. World Scientific.

Shull, A. G. (2012, April 17). U.S. Patent No. 8160783, Digging control system. Peoria, Ill: U.S. Patent and Trademark Office.

Singh, S. (1995). Synthesis of tactical plans for robotic excavation (PhD). Robotics Institute, Carnegie Mellon University, Pittsburgh, PA.

Spong, M., \& Vidyasagar, M. (1989). Robot dynamics and control. John Wiley and Sons.

Thangavelautham, J. (2008). A regulatory theory of cortical organization and its applications to robotics $(\mathrm{PhD})$. Institute for Aerospace Studies University of Toronto.

Zlotnikov, D. (2012, August). Mining in the extreme. CIM, 7(5), 50-56. 